\title{
Systematic Review on the Therapeutic Options for COVID-19: Clinical Evidence of Drug Efficacy and Implications
}

This article was published in the following Dove Press journal: Infection and Drug Resistance

\author{
Abdullahi Rabiu Abubakar (D) \\ Ibrahim Haruna Sani (D) ${ }^{2}$ \\ Brian Godman (iD) 3 \\ Santosh Kumar (iD ${ }^{4}$ \\ Salequl Islam (D) ${ }^{5}$ \\ Iffat Jahan iD ${ }^{6}$ \\ Mainul Haque iD $^{7}$ \\ 'Department of Pharmacology and \\ Therapeutics, Faculty of Pharmaceutical \\ Sciences, Bayero University, Kano, \\ Nigeria; ${ }^{2}$ Unit of Pharmacology, College \\ of Health Sciences, Yusuf Maitama Sule \\ University, Kano, Nigeria; ${ }^{3}$ Strathclyde \\ Institute of Pharmacy and Biomedical \\ Sciences, University of Strathclyde, \\ Glasgow, UK; ${ }^{4}$ Department of \\ Periodontology and Implantology, \\ Karnavati University, Gandhinagar \\ 382422, India; ${ }^{5}$ Department of \\ Microbiology, Jahangirnagar University, \\ Savar, Dhaka 1342, Bangladesh; \\ ${ }^{6}$ Department of Physiology, Eastern \\ Medical College, Cumilla, Bangladesh; \\ ${ }^{7}$ Unit of Pharmacology, Faculty of \\ Medicine and Defence Health, Universiti \\ Pertahanan Nasional Malaysia (National \\ Defence University of Malaysia), Kuala \\ Lumpur, 57000, Malaysia
}

\begin{abstract}
A novel coronavirus-2 (SARS-CoV-2) was first identified in Wuhan, China, and quickly spread globally. Several treatments have been proposed, many of which have proven ineffective. Consequently, there is a need to review the published evidence of drug clinical trials to guide future prescribing. A systematic review of published clinical trials and retrospective observational studies was carried out. The search was made using PubMed, Embase, MEDLINE, and China National Knowledge Infrastructure (CNKI) databases. Articles published between January 2020 and October 2020 and written in the English language were retrieved and included in the study. Researches that used traditional medicine, in-vitro and invivo animal studies, as well as reviews were excluded. Seventy-three relevant articles that fulfilled the inclusion criteria were finally selected and reviewed. Hydroxychloroquine, chloroquine, and azithromycin produced no clinical evidence of efficacy in randomized controlled clinical trials (RCT). However, retrospective observational studies reported the efficacy of remdesivir and lopinavir/ritonavir in reducing viral load, although there have been concerns with lopinavir/ritonavir and, more recently, remdesivir. Recently, tocilizumab, dexamethasone, and methylprednisolone significantly relieved lung inflammation and decreased mortality in patients with severe COVID-19. In addition, convalescent plasma was effective in boosting strong immunity among patients with mild COVID-19. There is currently no single worldwide approved therapeutic option for patients with COVID-19 despite the initial hype with medicines, including hydroxychloroquine. Nonetheless, dexamethasone has shown promise in symptomatic treatment and convalescent plasma in boosting immunity. New treatments are currently being researched, and the findings will be reported accordingly to provide evidence-based guidance for prescribers and policymakers. Keywords: COVID-19, efficacy, remdesivir, hydroxychloroquine, dexamethasone, lopinavir/ritonavir, clinical-trials, therapeutic-option
\end{abstract}

\section{Introduction}

In December 2019, several cases of mysterious pneumonia with the severe acute respiratory syndrome (SARS) were first reported in Wuhan, China. ${ }^{1,2}$. A novel coronavirus 2 (SARS-CoV-2) was identified as the cause and later designated as COVID-19 by the World Health Organization (WHO). ${ }^{2-5}$ The disease's main symptoms include fever, cough, shortness of breath, and fatigue. Other symptoms comprise sore throat, headache, myalgia, chills, nasal congestion, conjunctivitis, and diarrhea. ${ }^{2-6}$ Vital co-morbidities associated with death to COVID-19 include hypertension, diabetes, coronary heart disease, cerebral infarction, severe asthma,
Unit of Pharmacology, Faculty of Medicine and Defence Health, Universiti Pertahanan Nasional Malaysia (National Defence University of Malaysia), Kem Perdana Sungai Besi, Kuala Lumpur 57000, Malaysia

Tel + 60109265543

Email runurono@gmail.com 
pneumonia, and chronic bronchitis. ${ }^{5,7-13}$ In addition, death due to COVID-19 is associated with several disease symptoms. Prominent among them include chronic pneumonia, which causes severe respiratory distress, sepsis-associated organ failure, and high D-dimer levels, which indicate the high risk of deep vein thrombosis. Also, elderly patients may experience widespread inflammation and suppression of body defense mechanisms, making them vulnerable to opportunistic infections. ${ }^{5,14}$ Ethnicity may also play a role as seen, for instance, in the United Kingdom and the USA; however, the exact reasons underlying the early observations are uncertain. ${ }^{7,15,16}$ Furthermore, SARS-CoV-2 infection appeared to produce a lesser fatality rate $(3.1 \%)$ than previous SARS-CoV-1 (9.6\%) and the Middle East respiratory syndrome (MERS-CoV) (34.4\%). ${ }^{1,3,17}$ The novel SARS-CoV-2 is believed to invade a host's cell and initiate disease through binding to the angiotensinconverting enzyme-2 receptor (ACE-2 receptor). ${ }^{18-20}$ As such, an $A C E-2$ gene is considered responsible for coding genetic information for the expression of the ACE-2 receptor for both coronavirus 1 and $2 .^{18-21}$ Consequently, a higher expression of the ACE-2 receptors increases the risk of COVID-19 infections. Furthermore, some ACE-2 variants genes could reduce the association between ACE2 receptors and spike-protein of the coronavirus. Therefore, altering this gene could significantly affect the expression pattern of the human ACE-2 receptor in different cells and tissues, which appears critical for the susceptibility, symptoms, and outcome of COVID-19 infection. ${ }^{18,19}$ Patients on angiotensin-converting enzyme inhibitors (ACEI) or angiotensin receptor blockers (ARBs) are associated with a considerably low risk of COVID-19 infection. This is because ACEI stalls angiotensin's activity, converting enzymes linked to COVID-19 ACE-2 receptors, reducing the cell invasion. Equally, ARBs preoccupy the COVID-19 ACE-2 receptors, thus minimize the interaction between the coronavirus and the host's cells. Moreover, a report showed that COVID-19 patients on ACEI or ARBs had decreased the need for ICU admission. $^{22}$ However, the findings vary according to several issues, including the study population. ${ }^{23}$

A COVID-19 suspected patient is generally diagnosed using a reverse transcriptase-polymerase chain reaction (RT-PCR) assay, with a nasal swab or bronco-alveolar lavage fluid as test-specimens. ${ }^{24-26}$ Other methods of COVID-19 detection include radiologic imaging or chest $\mathrm{X}$-ray that are frequently employed to detect lung damage. Also, enzyme-linked immunoassays (ELISA), lateral flow immunoassays (LFIA), neutralization assays, and chemiluminescent assays are required COVID-19 testing methods. $^{24-26}$ COVID-19 patients are considered to have severe pneumonia, which may require a ventilator if they are experiencing respiratory distress $(\geq 30$ breaths per $\min )$; oxygen saturation at rest $(\leq 93 \%)$; a ratio of the partial pressure of arterial oxygen to fractional oxygen concentration $\left(\mathrm{paO}_{2} / \mathrm{fiO}_{2}\right) \leq 300 \mathrm{~mm} \mathrm{hg}$; or having severe complications such as organ failures. ${ }^{27,28}$ Lung inflammation, an essential symptom of severe pneumonia, occurs with baseline fever $\left(>38^{\circ} \mathrm{C}\right)$, C-reactive protein (10 times than usual $5 \mathrm{mg} / \mathrm{dl}$ ), ferritin (2 times of $400 \mu \mathrm{g} / \mathrm{l})$, or IL-6 (10 times than $3.4 \mathrm{mg} / \mathrm{l}) .^{27,28}$ The goal of managing patients with COVID-19 infection is to reduce the viral load, improve lung function, treat any fever, control diarrhea, and improve the body's immune system, known as the standard of care (SOC). ${ }^{27,28}$ Some medicines that have been prescribed for patients with COVID-19 include antiviral agents (lopinavir $40 \mathrm{mg} /$ ritonavir $100 \mathrm{mg}$ twice a day or remdesivir $100 \mathrm{mg}$ /day), antibiotics including azithromycin (500mg daily) and ceftriaxone (1g daily), antimalarials, including hydroxychloroquine (600mg daily), zinc sulfate (20mg daily), dexamethasone (6mg/day), and vita$\min$ C (3g daily). ${ }^{27,29-38}$

Recently, there have been concerns over several recommended medicines. Prominent among them is hydroxychloroquine (HCQ), with the concerns of lack of a control arm in the initial trials leading to the hype, adverse reactions, and suicides. ${ }^{30,31,39-43}$ Subsequent studies typically failed to show any clinical benefit from HCQ prescribing alongside potential harm resulting in the WHO dropping HCQ from its current global studies. $^{31,44-50}$ Lopinavir/ritonavir treatments have also been dropped by the WHO based on concerns of lack of efficacy. $^{44,51}$ This has prompted the need to try other potential medicines to treat patients with COVID-19. This is because preventative measures such as lockdowns, social distancing, frequent hand washing, track and trace systems, and closing of borders have resulted in unintended consequences. ${ }^{29,52-58}$ African countries, including Botswana, Ghana, Uganda, and Asian countries including Korea, Malaysia, and Vietnam, are currently having comparatively low infection prevalence and mortality rates than high-income countries, including Italy, Spain, the United Kingdom, and the USA. ${ }^{17,29,59-69}$ Sequel to those concerns mentioned earlier, this review will focus on published clinical trials and retrospective observational studies for the therapeutic options of COVID-19 infection. We are 
aware that there are several systematic and other reviews published already. ${ }^{31,33-35,37,46,70-84}$ However, we intended to build on this and analyze the most recent published papers to summarize some evidence-based therapeutic options for COVID-19 infection.

\section{Materials and Methods Data Sources}

Data sources were four electronic databases, including PubMed, Embase, MEDLINE, and CNKI. The search terms used were clinical trials, evidence of efficacy, COVID-19, drug treatment, randomized, double-blinded, controlled trials, retrospective study, and therapeutic options.

\section{Study Selection}

The study was conducted according to the Prisma methodology for preferred reporting items for systematic reviews and meta-analyses, protocols 2015 (Prisma-p 2015). ${ }^{85}$ Initially, 427 articles were retrieved from the databases on the therapeutic options for COVID-19 independently by the first two authors. After collecting the retrieved articles together, 136 duplicates were excluded. In addition, 183 articles were either in-vitro, in-vivo animal studies, or used medicinal plants, subsequently removed from collections. Afterward, 35 more articles were further excluded because they were either review or did not utilize an appropriate study design. Finally, 73 published articles that met the inclusion criteria were appraised in depth. This systematic review has included pre-printed publications where relevant as research on the COVID-19 Pandemic is a novel and evolving research area. The article retrieval, screening, and inclusion flow chart are shown in Figure 1.

\section{Inclusion Criteria}

Studies published between January 2020 and October 2020 and written in the English language.

\section{Exclusion Criteria}

Studies undertaken using medicinal plants, in-vitro studies, in-vivo animal studies, and reviews.

\section{Data Extraction}

Published clinical trials and retrospective observational studies were selected. A further search was made to verify the articles' clinical trial registration number at www.clin icaltrials.gov, European clinical trial registry at https:// www.clinicaltrialsregister.eu/, and Chinese clinical trial registry at http://www.chictr.org.cn/searchprojen.aspx. The reference section of all the articles reviewed was used to generate additional relevant publications. There was also no attempt to assess the published papers' quality using scales such as the Newcastle Ottawa scale as some key papers are likely to be pre-publication. ${ }^{86-89}$

\section{Results}

Our findings revealed a high degree of therapeutic failure among most of the medicines studied, building on recent NIH and WHO deliberations. The findings will be broken down by pertinent medicines starting with HCQ, with prospective and retrospective observational studies combined. Full details were depicted in Tables 1 and 2 .

\section{Hydroxychloroquine (HCQ) with and without Azithromycin (AZM)}

Hydroxychloroquine does not bind directly to the ACE-2 receptor for COVID-19 but acts by increasing the medium's acidity around which COVID-19 protein-spikes interact and bind ACE- 2 receptors. The acidity will make the medium hash, thereby degrading the viral spike and reducing the infection rate and spread of COVID-19. ${ }^{90}$ Azithromycin directly blocks the binding of COVID-19 virus with ACE-2 receptors on the host's cell surface. Consequently, HCQ and AZM may have a place in the prophylaxis and treat patients with mild-moderate COVID-19 infections. ${ }^{90}$ However, randomized controlled clinical trials (RCT) conducted in the United States and parts of Canada reported therapeutic failure of HCQ among 1309 COVID-19 asymptomatic patients. ${ }^{91}$ Similarly, HCQ did not produce better clinical outcomes than placebo among 30 treatment-naive patients in China. ${ }^{92}$ In addition, HCQ failed to effectively treat 423 COVID-19 patients in a randomized, double-blind, placebo-controlled clinical trial conducted in Canada and the USA $^{93}$ Other studies revealed therapeutic failure among 181 patients admitted to four different tertiary healthcare centers in France. ${ }^{94}$ Alongside this, therapeutic failure was also seen among 293 patients with mild COVID-19 versus usual care. ${ }^{95}$ Furthermore, HCQ, when combined with AZM, did not reduce mortality or the need for mechanical ventilation among COVID-19 patients enrolled in a randomized, controlled clinical trial in the USA. $^{96}$ Equally, HCQ combined with AZM did not 


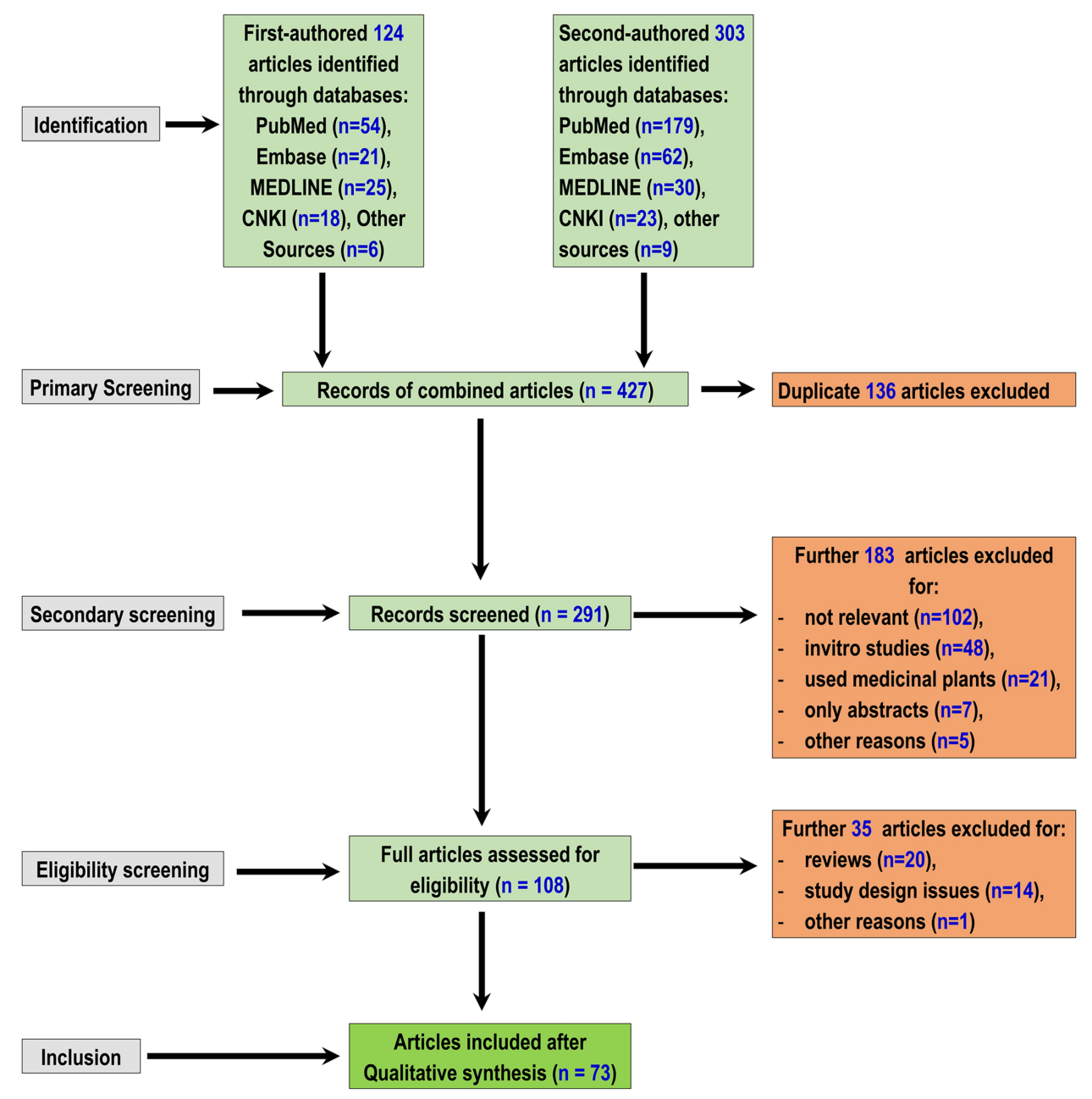

Figure I A Flow Chart Illustrating the Selection Process of Manuscripts.

produce better clinical outcomes among 504 COVID-19 patients versus standard care. ${ }^{97}$ The UK recovery study involving 1542 patients randomized to HCQ compared with 3132 patients randomized to usual care showed no significant difference in 28-day mortality, duration of hospital stay, the need for mechanical ventilation, renal replacement, and development of major cardiac arrhythmias. ${ }^{98}$

In an observational study involving 1376 consecutive patients in the USA, Geleris et al (2020) found that HCQ did not improve the outcomes using an endpoint of intubation of death versus non-HCQ patients. ${ }^{99}$ In a large cohort study in the USA involving 3372 patients hospitalized with COVID-19, Singh et al (2020) demonstrated that HCQ did not confer any benefits to patients versus those who did not receive HCQ. ${ }^{100}$ Ip et al (2020) in their study conducted in the USA involving 2512 patients also found no differences in mortality in patients prescribed HCQ with or without AZM versus patients receiving neither therapy. ${ }^{101}$ In the treatment of COVID-19 using HCQ with or without AZM, patients' safety has been of great concern amplified by suicide attempts. ${ }^{41,102,103}$ In line with this, recent reviews indicated that HCQ causes neuropsychiatric side effects such as depression, psychosis, insomnia, manic episodes, and increased risk of suicide. ${ }^{104,105} \mathrm{In}$ addition, more studies have reported adverse drug reactions associated with HCQ, especially prolongation of QTc (Corrected QT Interval). The QT interval is the time from the beginning of the QRS complex, representing ventricular depolarization, to the end of the $\mathrm{T}$ wave, resulting from the ventricular repolarization interval. ${ }^{48,49,91,106}$ However, Mahévas et al (2020) found that only $10 \%$ of patients experience electrocardiographic modifications requiring discontinuation of therapy ${ }^{94}$ Furtado et al (2020) found no therapeutic benefit in their open-label randomized trial involving 447 patients from adding AZM to HCQ. ${ }^{107}$ Some of these findings might be explained because HCQ 


\begin{tabular}{|c|c|c|c|c|c|c|c|c|}
\hline 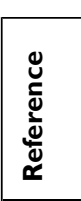 & 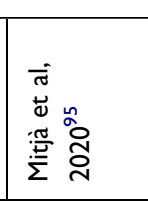 & 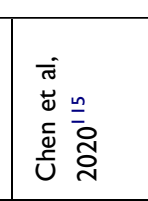 & 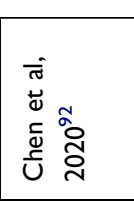 & 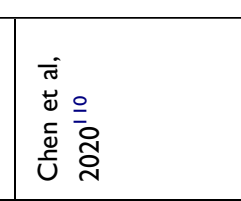 & 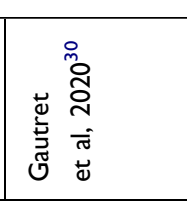 & 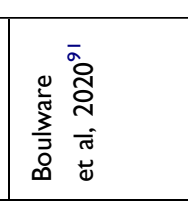 & 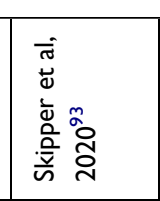 & 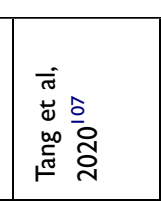 \\
\hline 兽 & 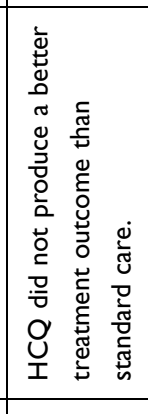 & 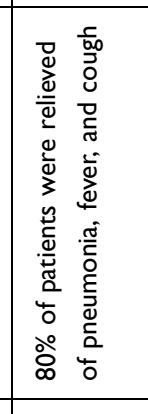 & 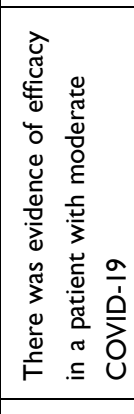 & 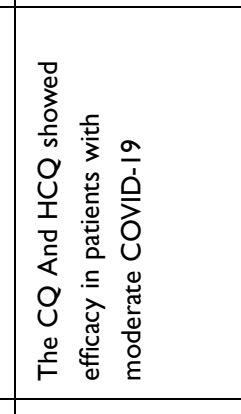 & 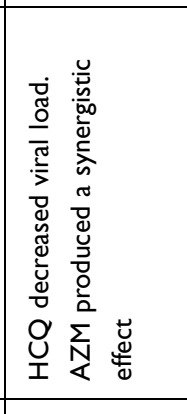 & 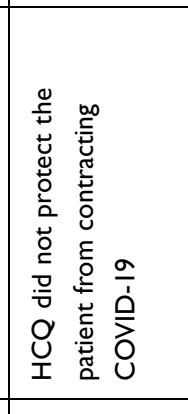 & 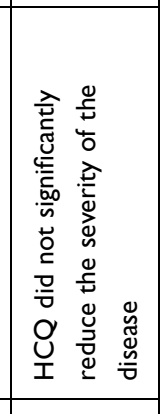 & 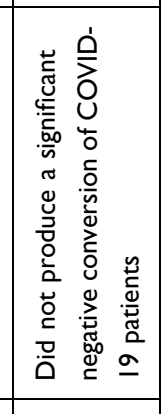 \\
\hline 高 & 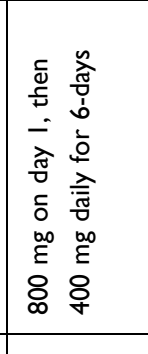 & 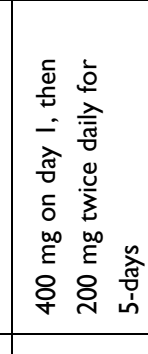 & 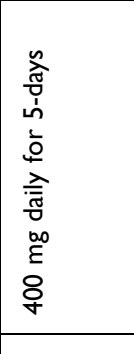 & 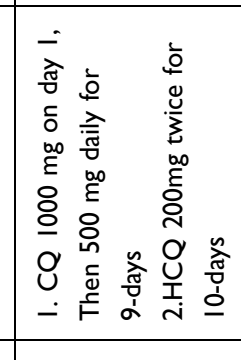 & 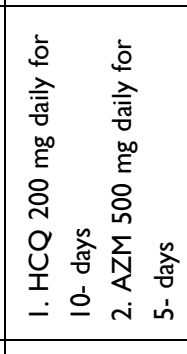 & 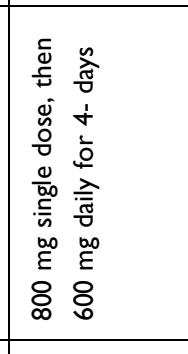 & 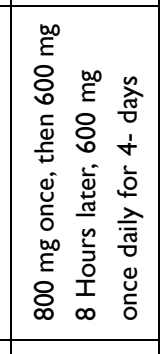 & 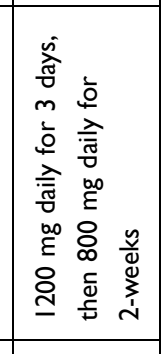 \\
\hline 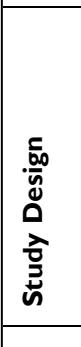 & 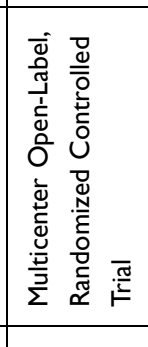 & 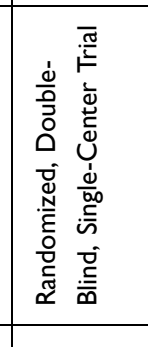 & 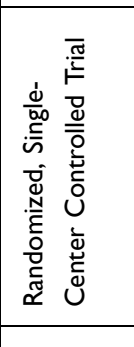 & 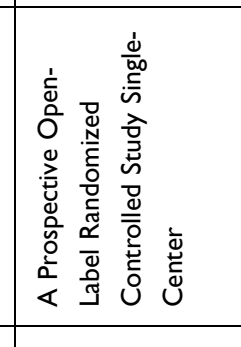 & 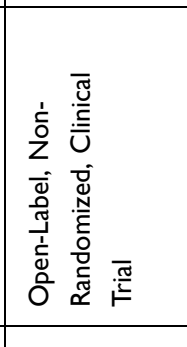 & 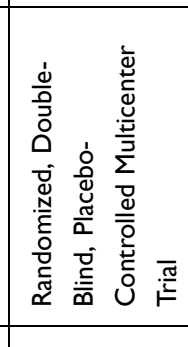 & 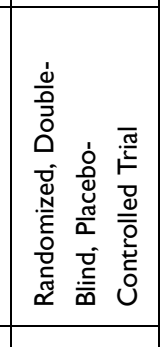 & 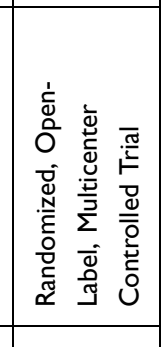 \\
\hline 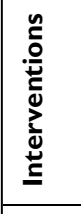 & \begin{tabular}{|l}
$\stackrel{q}{x}$ \\
I
\end{tabular} & $\stackrel{\stackrel{g}{I}}{\underline{I}}$ & $\stackrel{g}{\underline{I}}$ & 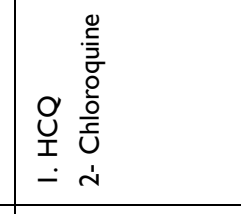 & 量离 & $\begin{array}{l}\stackrel{g}{I} \\
\end{array}$ & $\begin{array}{l}\stackrel{g}{x} \\
\end{array}$ & $\stackrel{\stackrel{g}{I}}{\underline{I}}$ \\
\hline 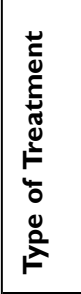 & 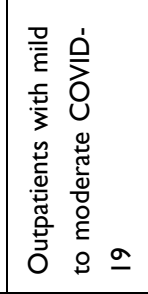 & 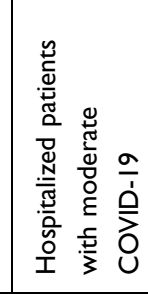 & 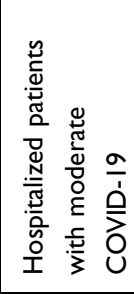 & 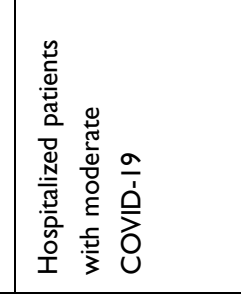 & 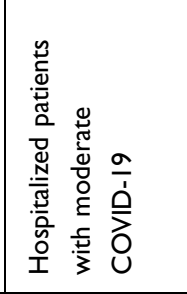 & 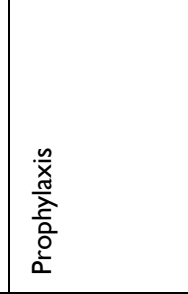 & 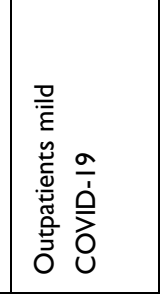 & 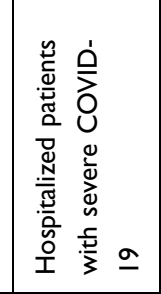 \\
\hline 䋨 & $\approx$ & $\approx$ & $\stackrel{\circ}{\circ}$ & 5 & F & 总 & $\approx$ & 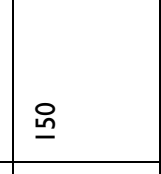 \\
\hline & & 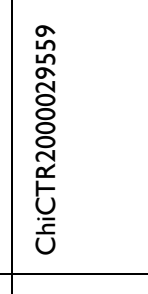 & 耪 & 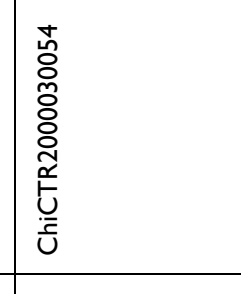 & 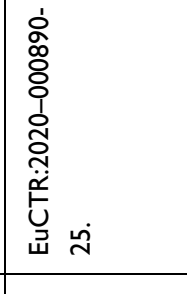 & 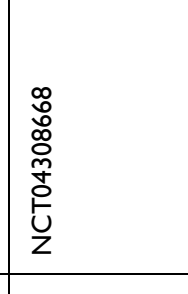 & 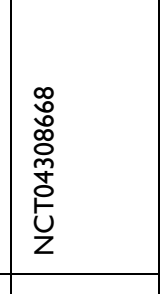 & 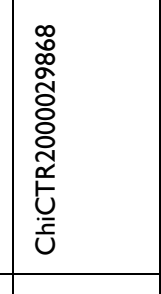 \\
\hline
\end{tabular}




\begin{tabular}{|c|c|c|c|c|c|c|c|c|}
\hline 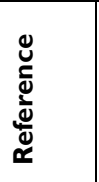 & 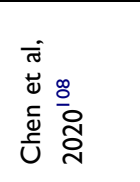 & 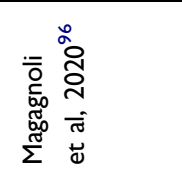 & 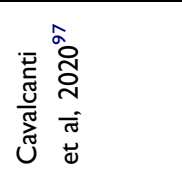 & 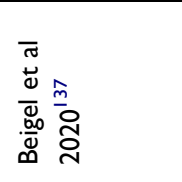 & 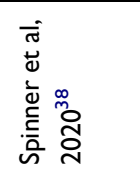 & 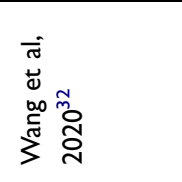 & 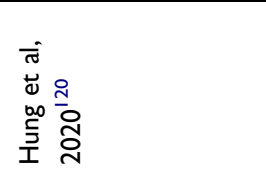 & 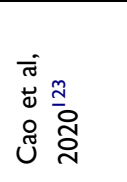 \\
\hline 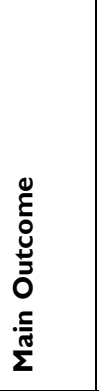 & 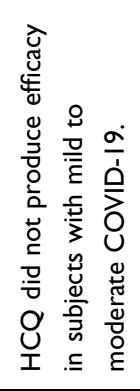 & 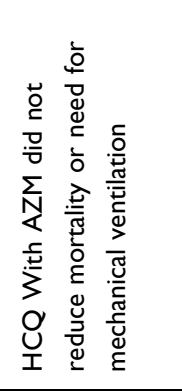 & 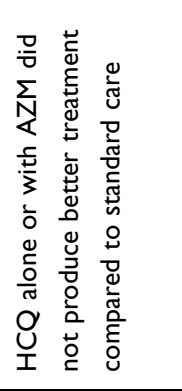 & 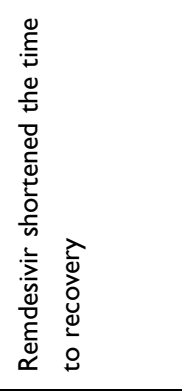 & 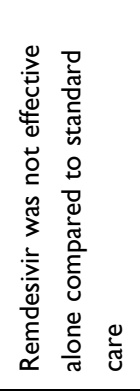 & 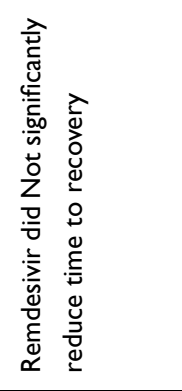 & 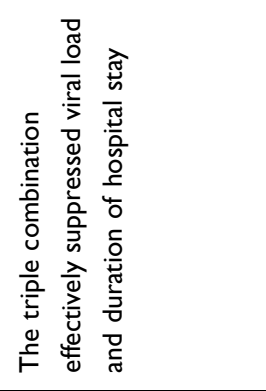 & 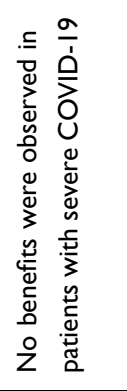 \\
\hline $\begin{array}{l}y \\
\vdots \\
\vdots\end{array}$ & 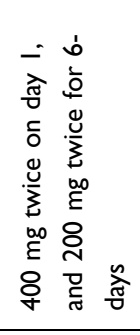 & 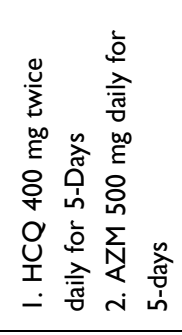 & 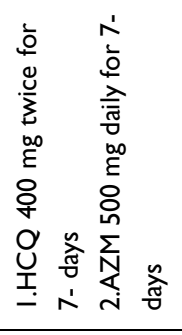 & 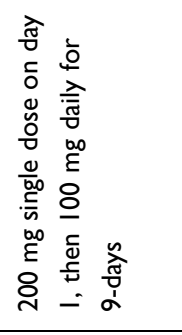 & 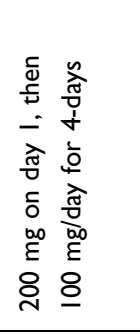 & 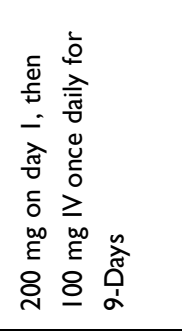 & 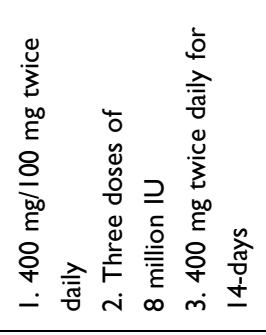 & 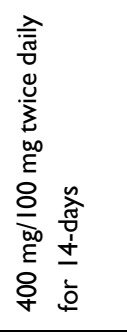 \\
\hline 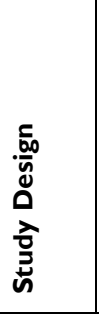 & 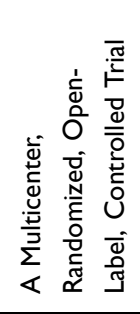 & 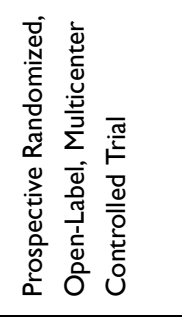 & 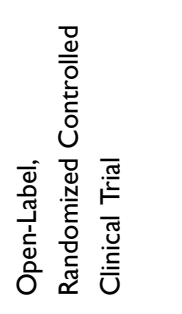 & 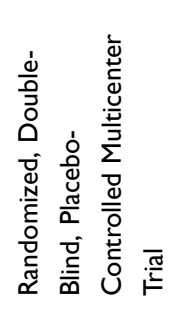 & 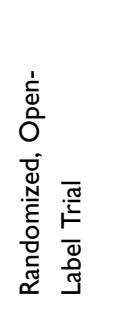 & 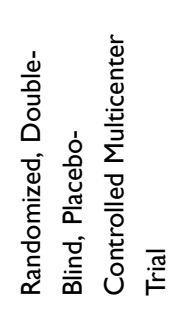 & 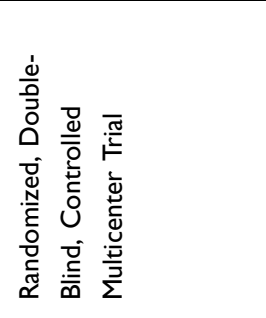 & 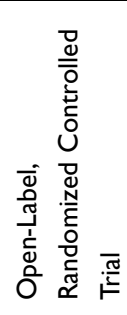 \\
\hline 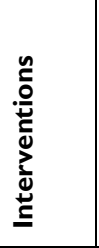 & $\underset{\mathrm{U}}{\mathrm{O}}$ & $\begin{array}{ll}0 & \sum \\
U & N \\
I & \mathbb{\alpha} \\
- & i\end{array}$ & 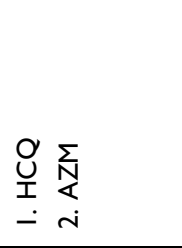 & 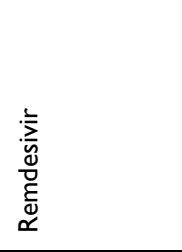 & 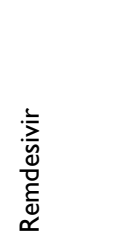 & 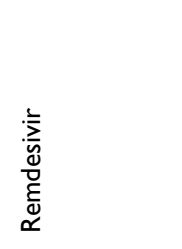 & 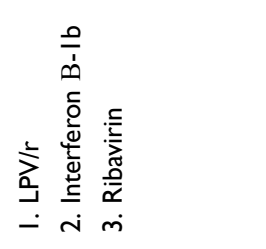 & $\stackrel{1}{a}$ \\
\hline 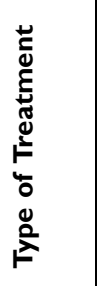 & 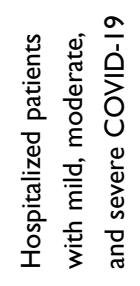 & 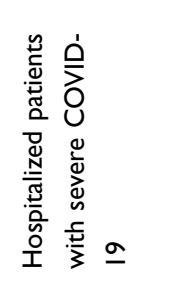 & 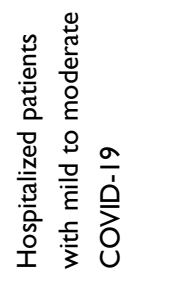 & 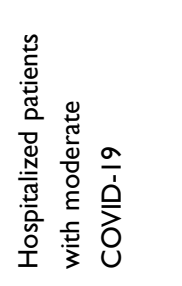 & 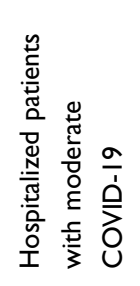 & 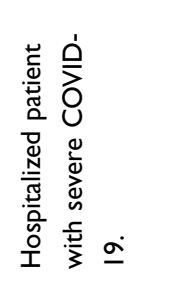 & 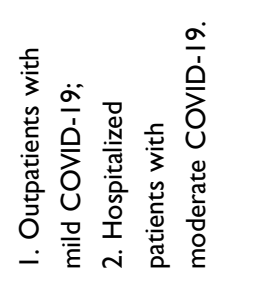 & 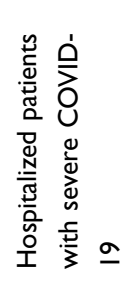 \\
\hline 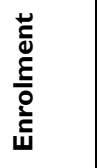 & $\stackrel{m}{m}$ & 今े & ثั & 음 & 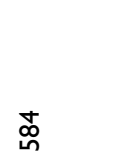 & $\hat{\sim}$ & $\infty$ & $\stackrel{\alpha}{\alpha}$ \\
\hline 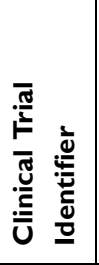 & 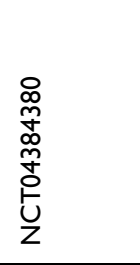 & 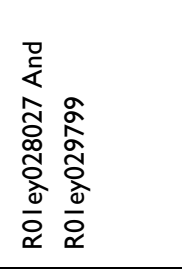 & 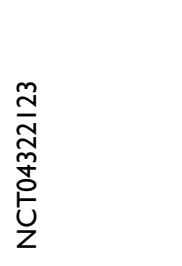 & 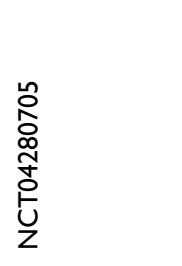 & 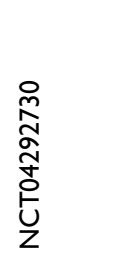 & 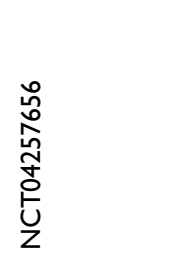 & 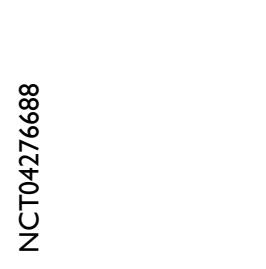 & 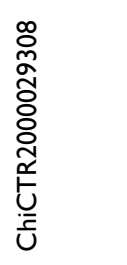 \\
\hline $\bar{n} \dot{z}$ & & & & & & & & \\
\hline
\end{tabular}




\begin{tabular}{|c|c|c|c|c|c|c|c|c|}
\hline 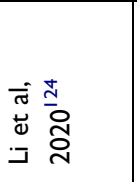 & 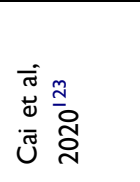 & 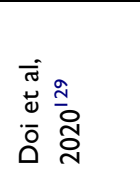 & 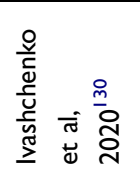 & 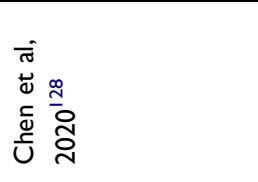 & 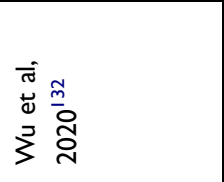 & 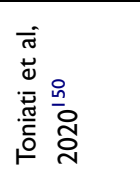 & 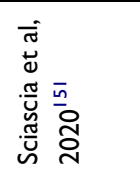 & 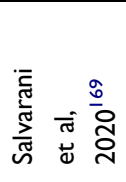 \\
\hline 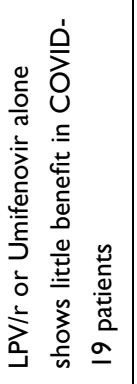 & 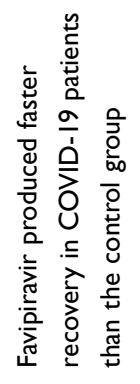 & 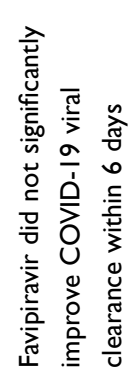 & 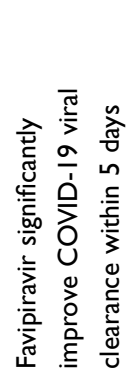 & 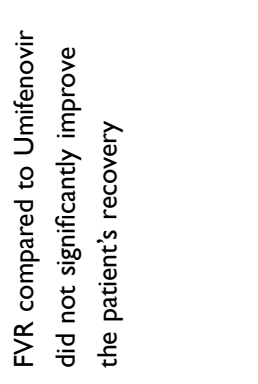 & 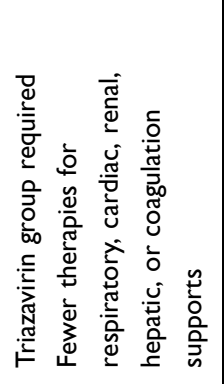 & 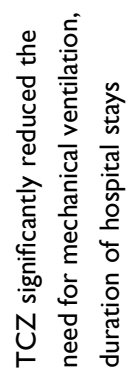 & 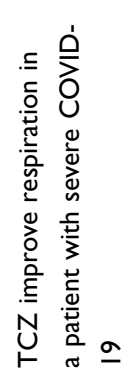 & 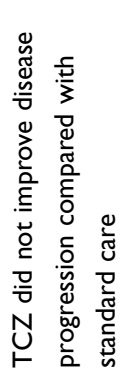 \\
\hline 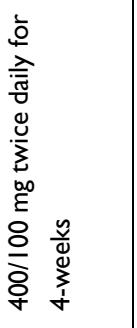 & 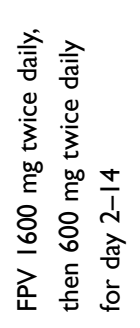 & 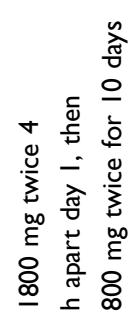 & 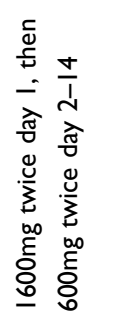 & 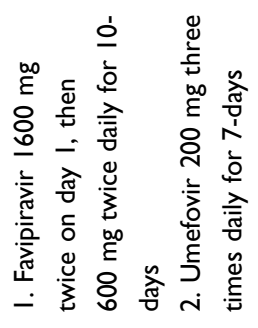 & 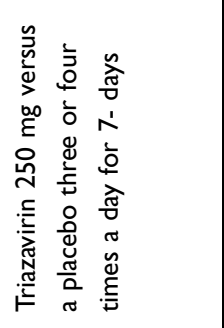 & 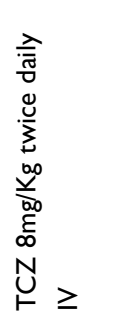 & 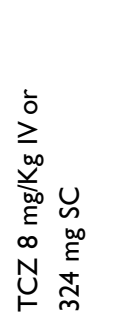 & 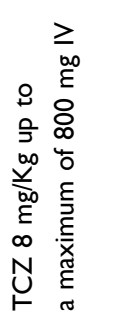 \\
\hline 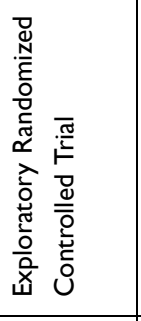 & 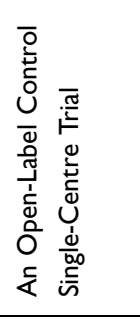 & 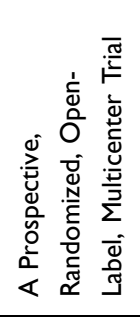 & 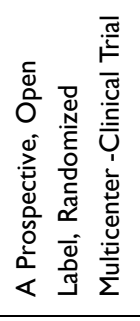 & 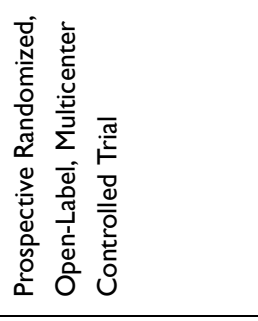 & 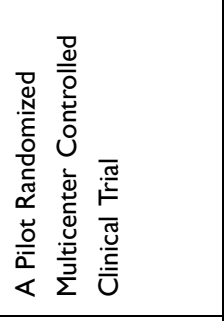 & 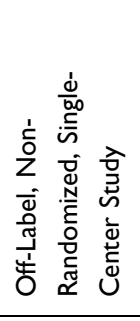 & 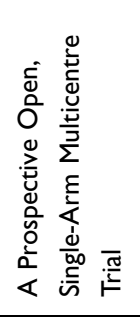 & 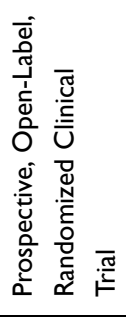 \\
\hline 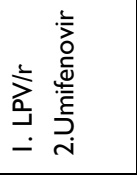 & 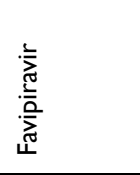 & 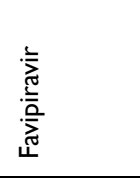 & 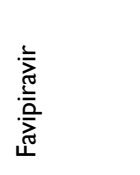 & 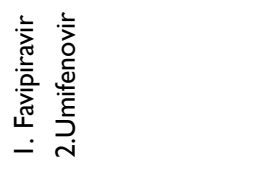 & 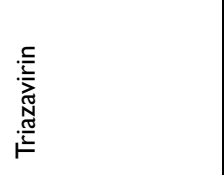 & $\underset{\vdash}{N}$ & $\underset{\vdash}{N}$ & $\underset{\vdash}{N}$ \\
\hline 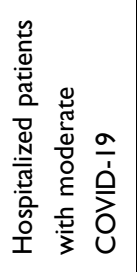 & 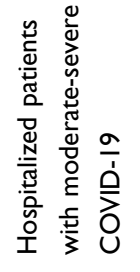 & 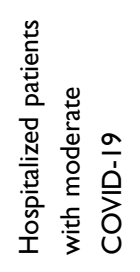 & 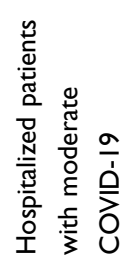 & 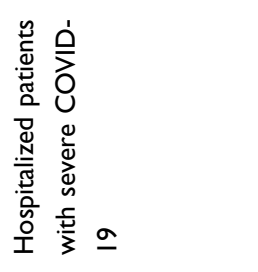 & 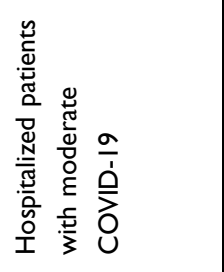 & 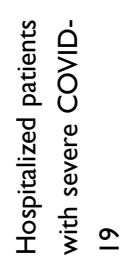 & 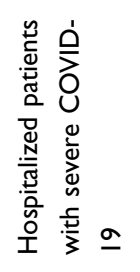 & 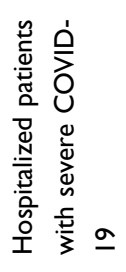 \\
\hline œ & ঃ & ó & ㅇ & $\stackrel{+}{\sim}$ & กี & 음 & $\tilde{0}$ & $\stackrel{\text { I }}{\simeq}$ \\
\hline 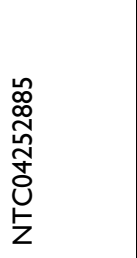 & 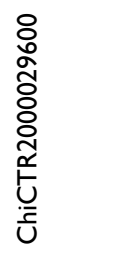 & 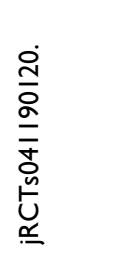 & 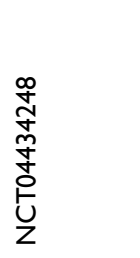 & 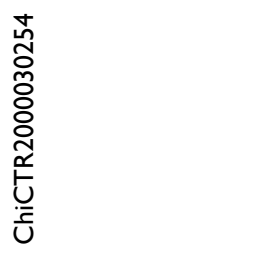 & 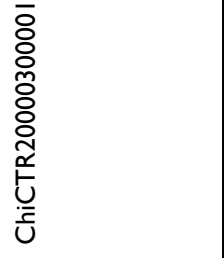 & 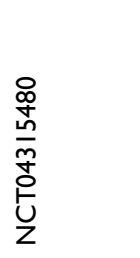 & & 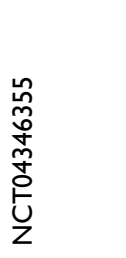 \\
\hline
\end{tabular}




\begin{tabular}{|c|c|c|c|c|c|}
\hline 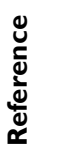 & 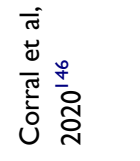 & 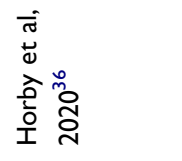 & 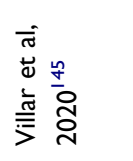 & 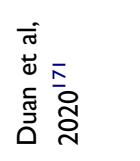 & 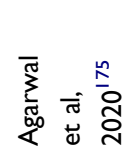 \\
\hline 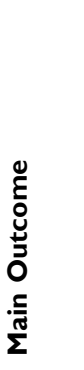 & 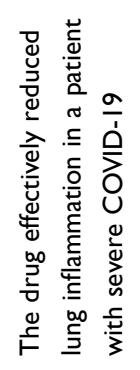 & 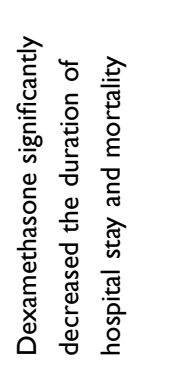 & 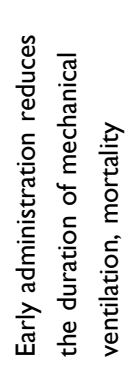 & 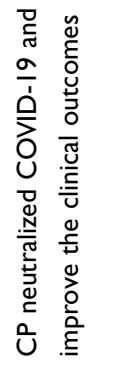 & 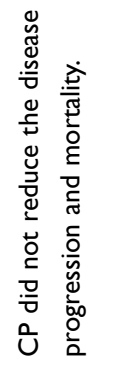 \\
\hline 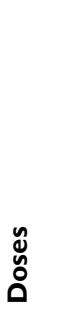 & 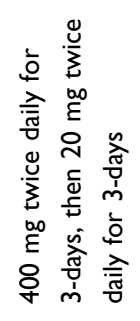 & 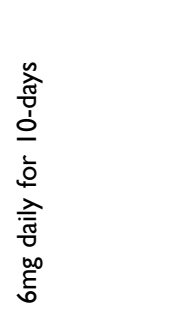 & 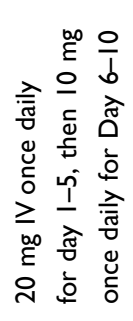 & 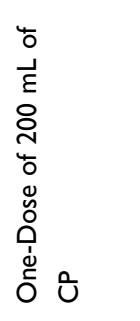 & 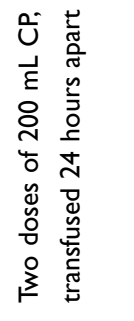 \\
\hline 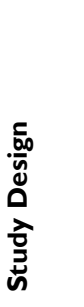 & 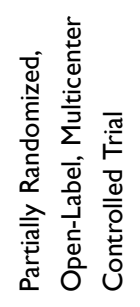 & 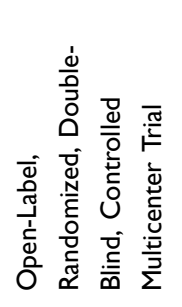 & 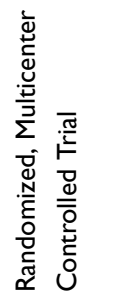 & 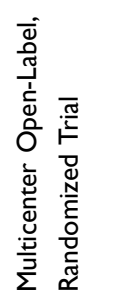 & 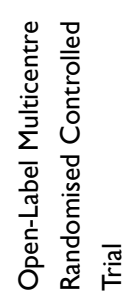 \\
\hline 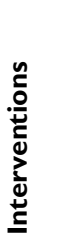 & 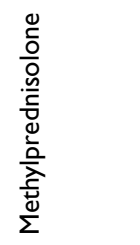 & 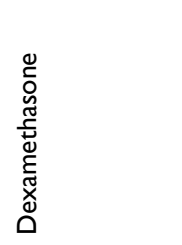 & 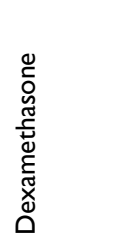 & 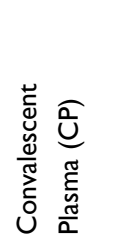 & 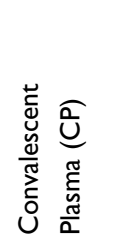 \\
\hline 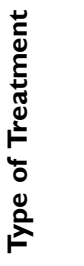 & 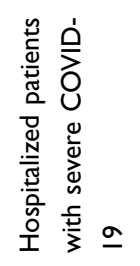 & 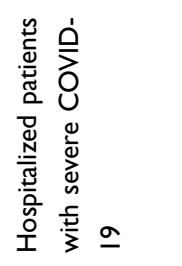 & 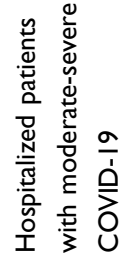 & 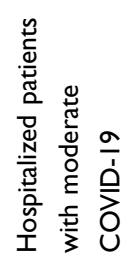 & 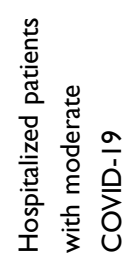 \\
\hline 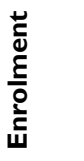 & $\stackrel{\text { 岗 }}{ }$ & 岂 & స & 으 & 志 \\
\hline 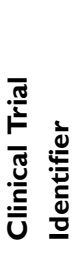 & 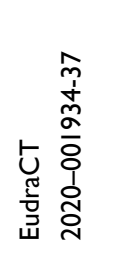 & 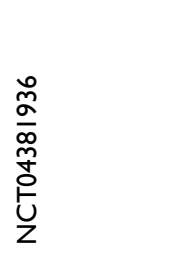 & 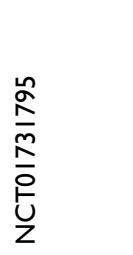 & 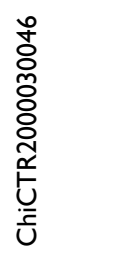 & 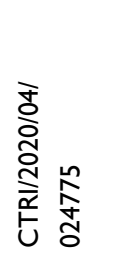 \\
\hline $\bar{n} \dot{z}$ & & & & & \\
\hline
\end{tabular}




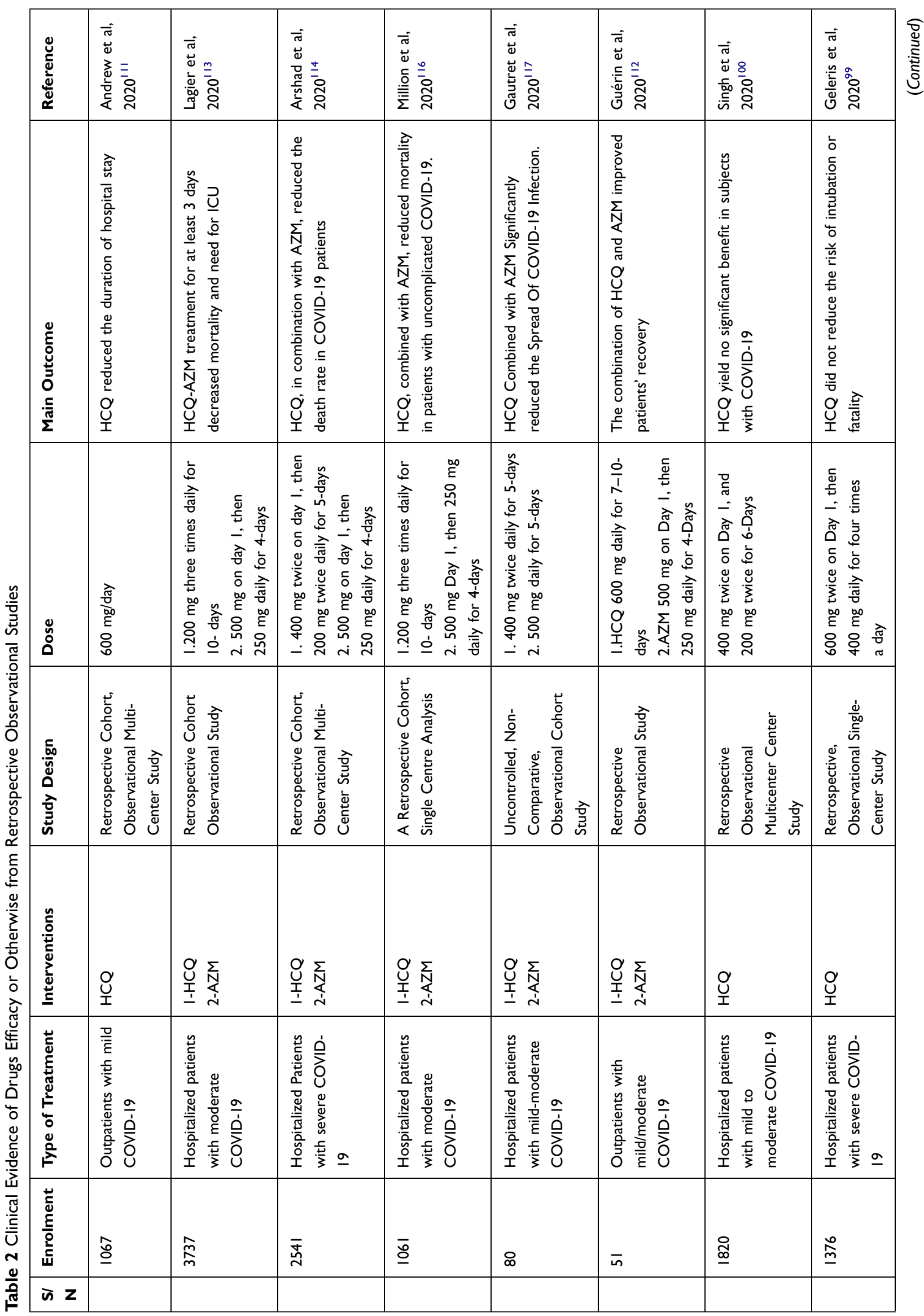




\begin{tabular}{|c|c|c|c|c|c|c|c|c|c|}
\hline 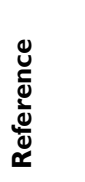 & 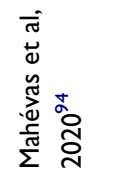 & 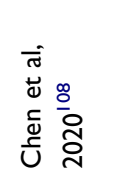 & 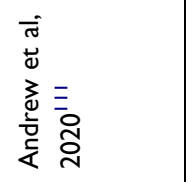 & 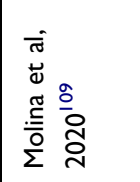 & 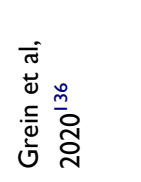 & 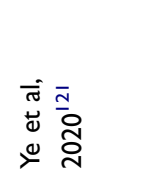 & 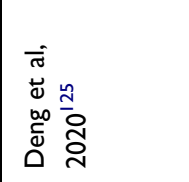 & 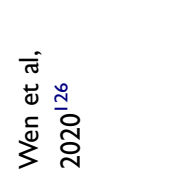 & 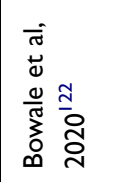 \\
\hline 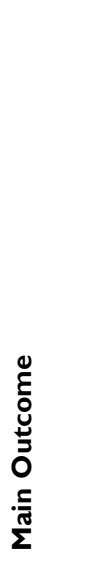 & 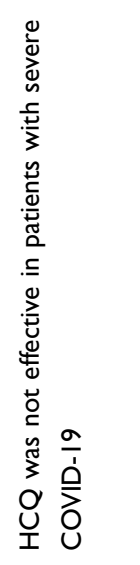 & 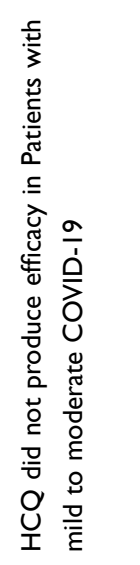 & 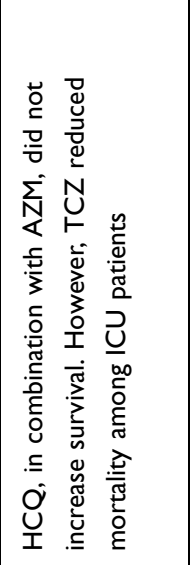 & 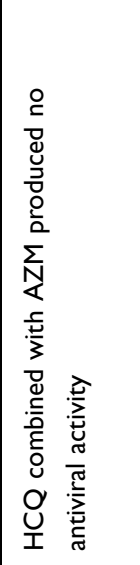 & 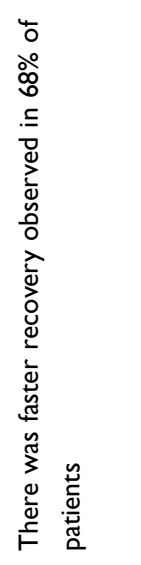 & 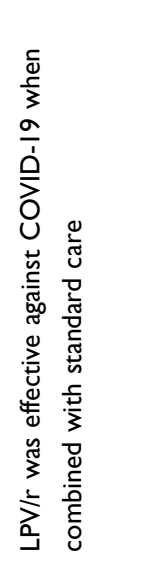 & 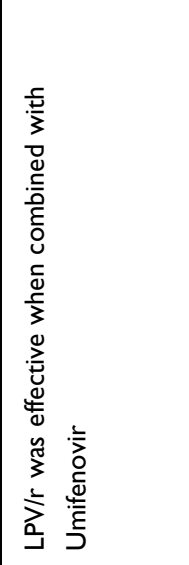 & 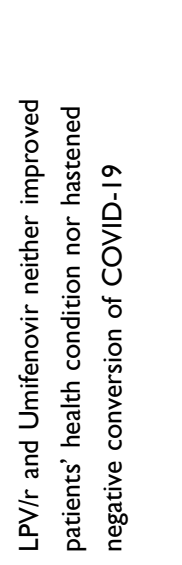 & 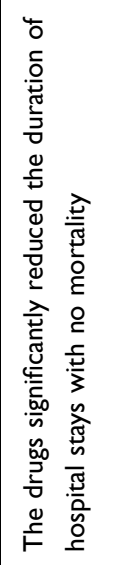 \\
\hline ڤั & $\begin{array}{l}\text { त् } \\
\stackrel{0}{\mathrm{~b}} \\
\varepsilon \\
\stackrel{0}{0} \\
0\end{array}$ & 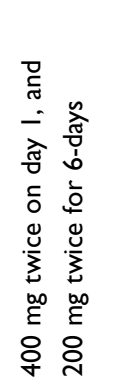 & 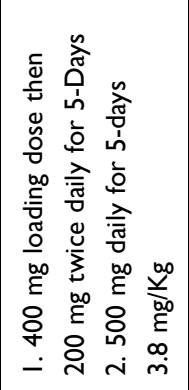 & 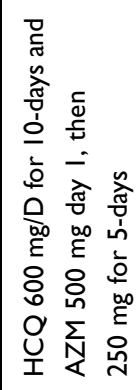 & 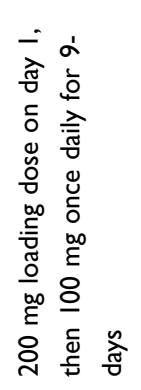 & 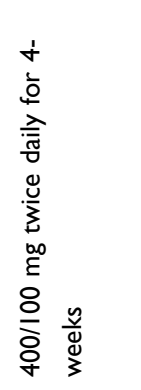 & 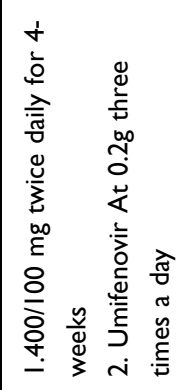 & 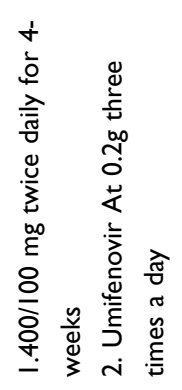 & 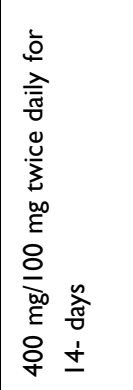 \\
\hline 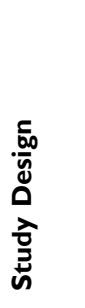 & 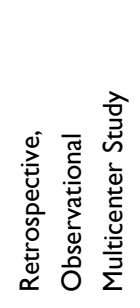 & 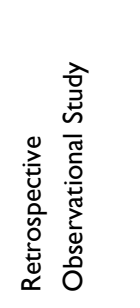 & 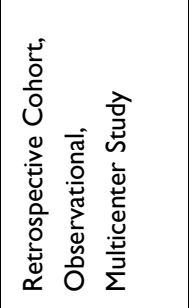 & 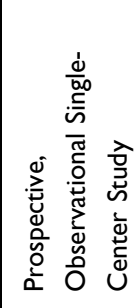 & 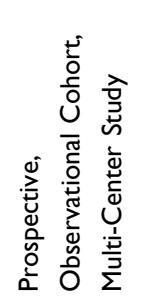 & 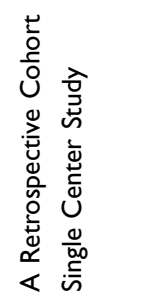 & 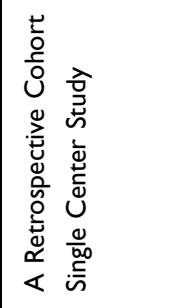 & 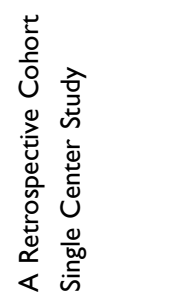 & 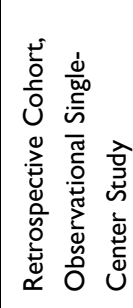 \\
\hline 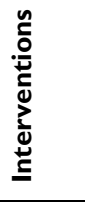 & 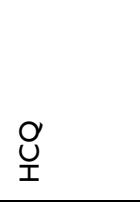 & $\underset{\text { Ư }}{O}$ & 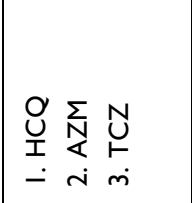 & 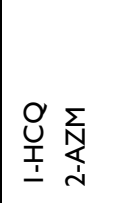 & 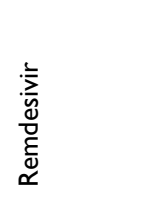 & $\stackrel{+}{\perp}$ & 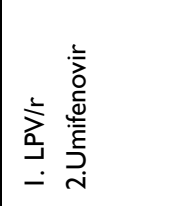 & 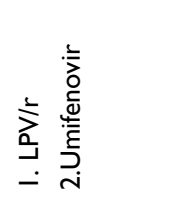 & 高 \\
\hline 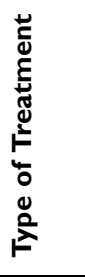 & 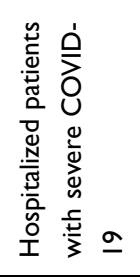 & 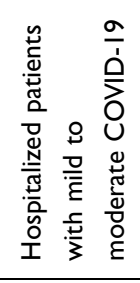 & 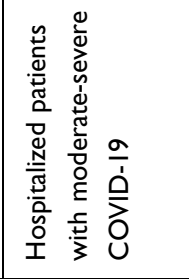 & 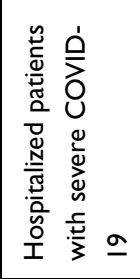 & 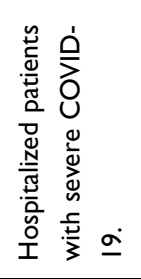 & 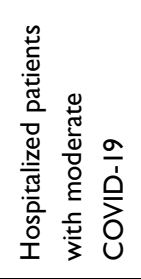 & 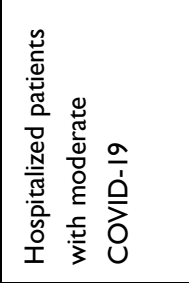 & 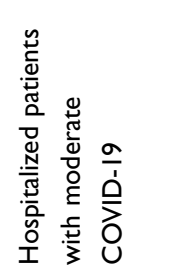 & 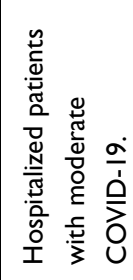 \\
\hline 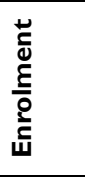 & $\underline{\bar{\infty}}$ & $\hat{m}$ & ஸे & $=$ & $\overline{0}$ & $\hat{f}$ & m & $\stackrel{\infty}{\underline{\infty}}$ & $\stackrel{\sim}{m}$ \\
\hline $\bar{n} z$ & & & & & & & & & \\
\hline
\end{tabular}




\begin{tabular}{|c|c|c|c|c|c|c|c|c|c|}
\hline 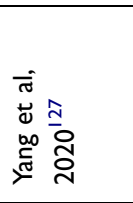 & 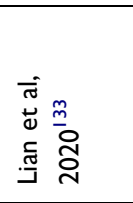 & 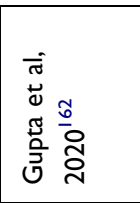 & 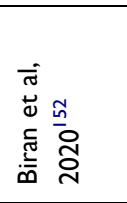 & 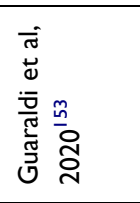 & 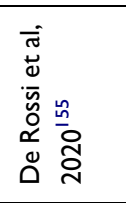 & 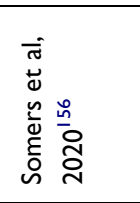 & 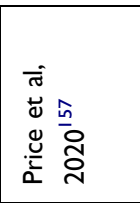 & 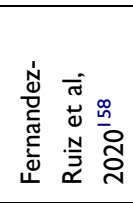 & 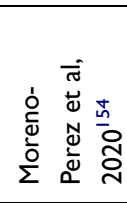 \\
\hline 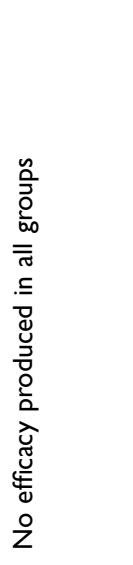 & 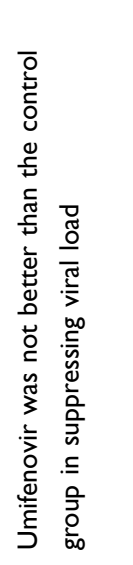 & 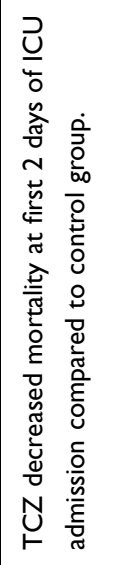 & 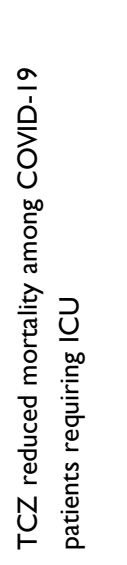 & 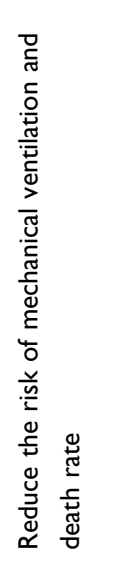 & 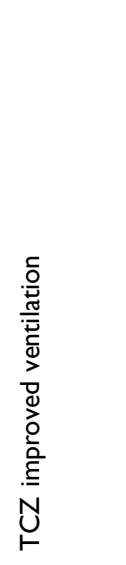 & 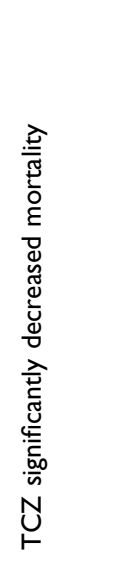 & 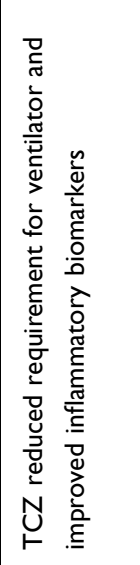 & 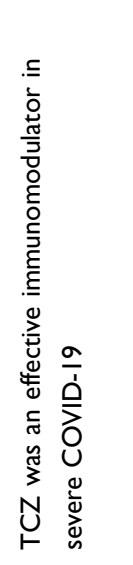 & 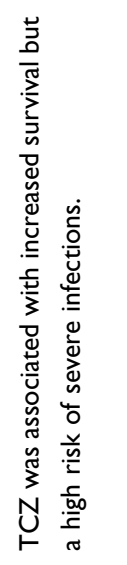 \\
\hline 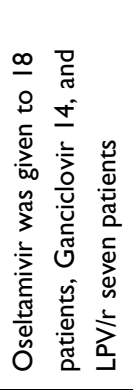 & 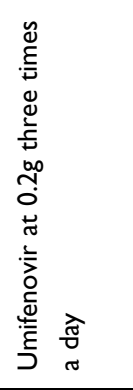 & 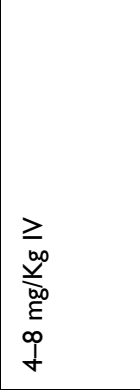 & 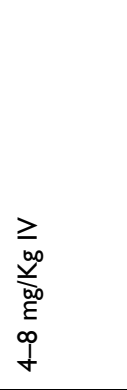 & 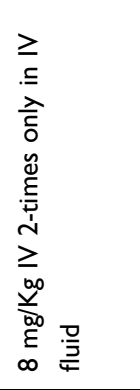 & 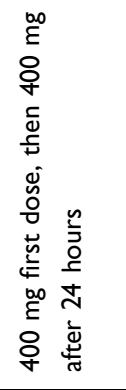 & 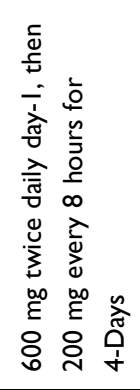 & 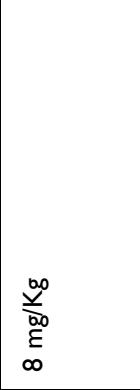 & 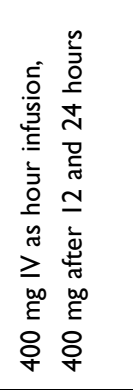 & 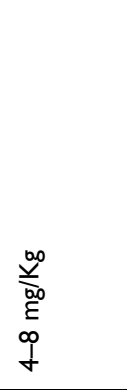 \\
\hline 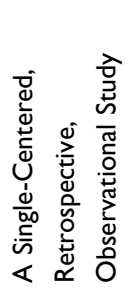 & 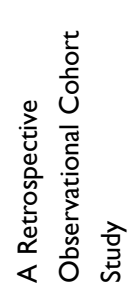 & 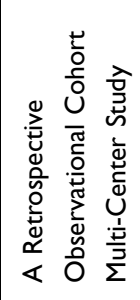 & 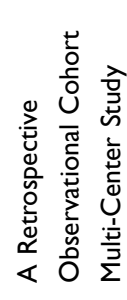 & 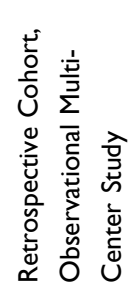 & 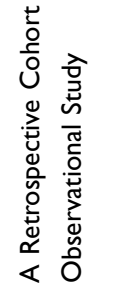 & 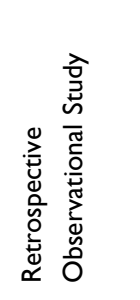 & 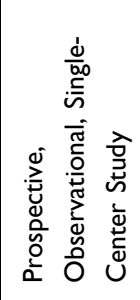 & 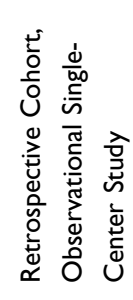 & 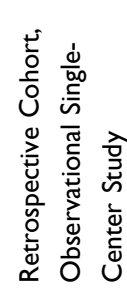 \\
\hline 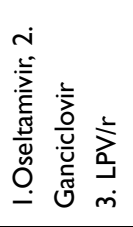 & 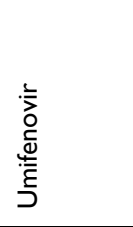 & $\underset{\cup}{N}$ & $\underset{\vdash}{N}$ & $\underset{\vdash}{N}$ & $\underset{\vdash}{N}$ & $\underset{\vdash}{N}$ & $\underset{\vdash}{N}$ & $\underset{\vdash}{N}$ & $\underset{\vdash}{N}$ \\
\hline 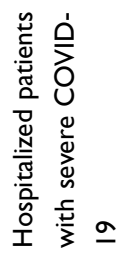 & 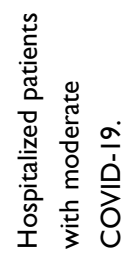 & 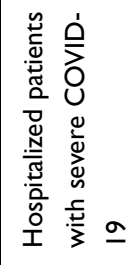 & 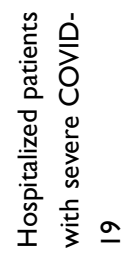 & 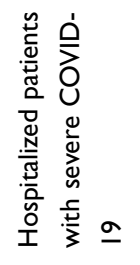 & 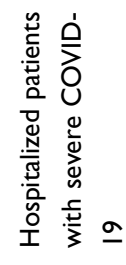 & 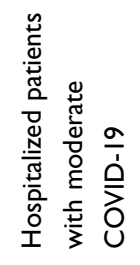 & 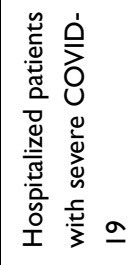 & 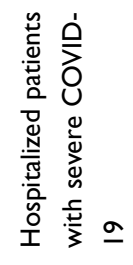 & 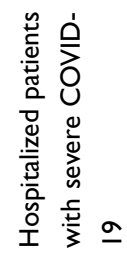 \\
\hline กิ & $\bar{\infty}$ & $\underset{j}{\sigma}$ & ర్లి & में & $\stackrel{\stackrel{\infty}{\varrho}}{\underline{n}}$ & 兽 & O & $\stackrel{\infty}{\infty}$ & 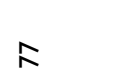 \\
\hline
\end{tabular}




\begin{tabular}{|c|c|c|c|c|c|c|c|c|c|c|}
\hline 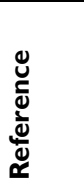 & 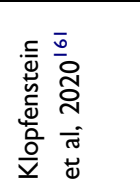 & 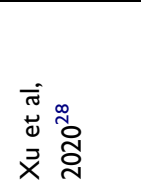 & 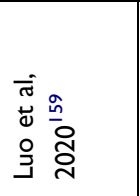 & 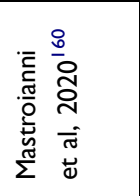 & 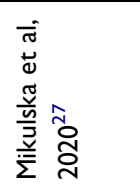 & 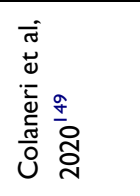 & 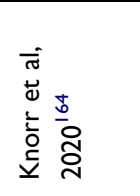 & 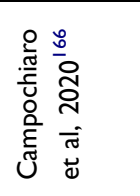 & 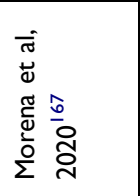 & 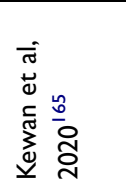 \\
\hline 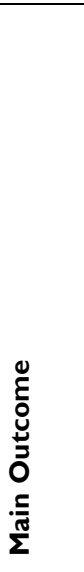 & 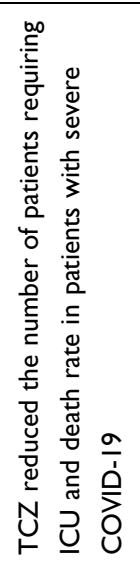 & 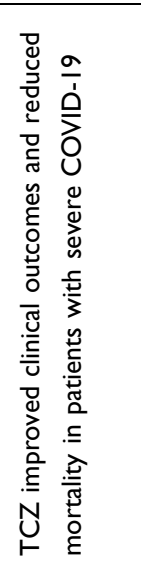 & 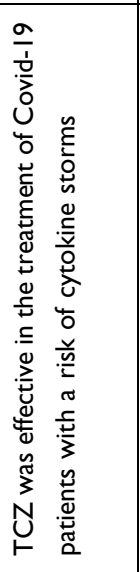 & 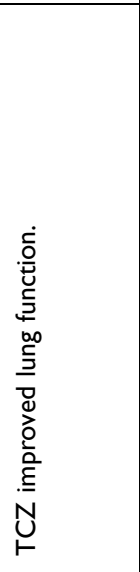 & 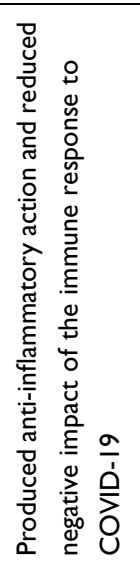 & 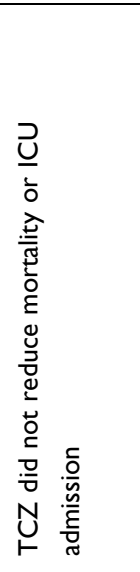 & 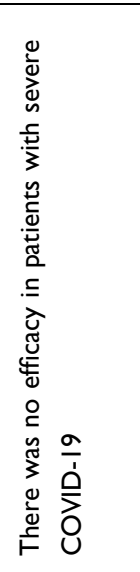 & 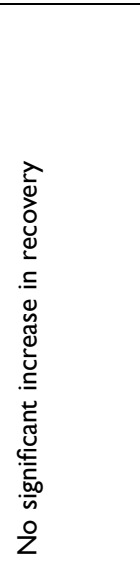 & 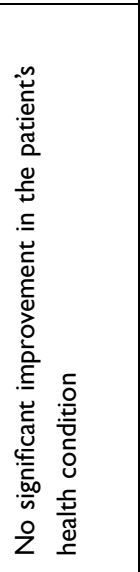 & 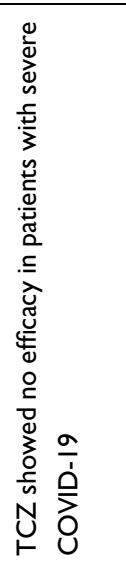 \\
\hline $\begin{array}{l}\stackrel{\Delta}{~} \\
\stackrel{\Delta}{\Delta}\end{array}$ & 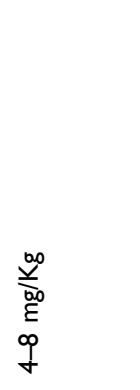 & 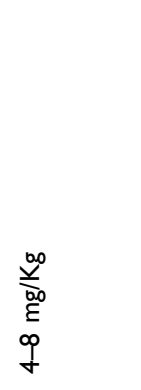 & 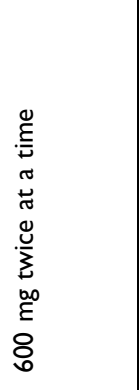 & 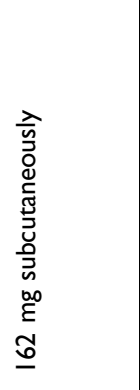 & 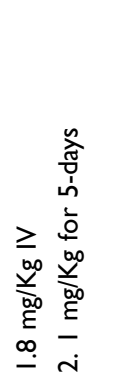 & 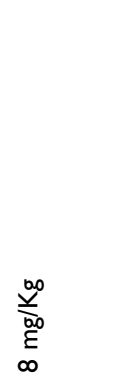 & 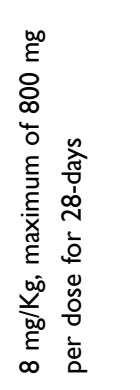 & 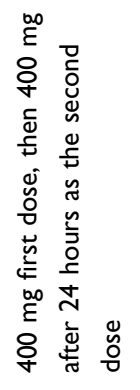 & 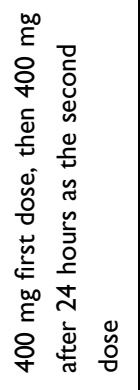 & 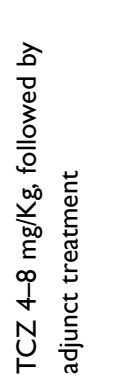 \\
\hline 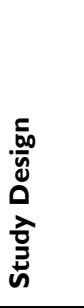 & 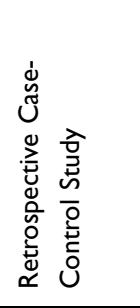 & 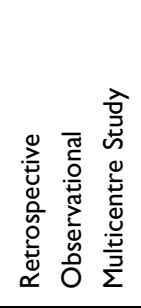 & 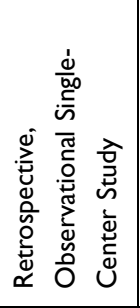 & 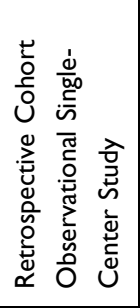 & 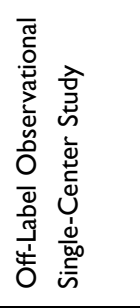 & 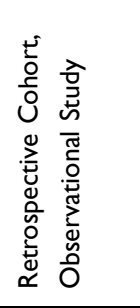 & 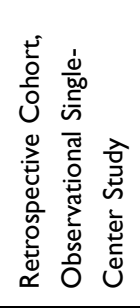 & 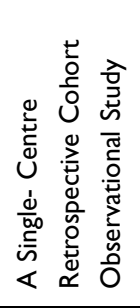 & 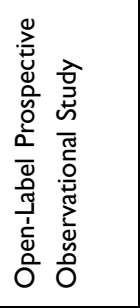 & 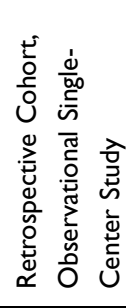 \\
\hline 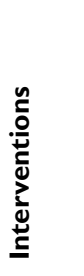 & $\underset{\vdash}{N}$ & $\underset{\vdash}{N}$ & $\underset{\vdash}{N}$ & $\underset{\vdash}{N}$ & 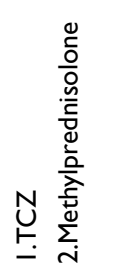 & $\underset{\vdash}{N}$ & $\underset{\vdash}{N}$ & $\underset{\vdash}{N}$ & $\underset{\vdash}{N}$ & $\underset{\vdash}{N}$ \\
\hline 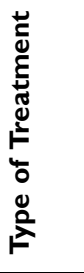 & 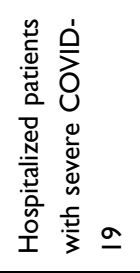 & 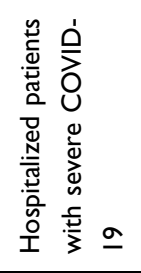 & 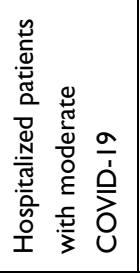 & 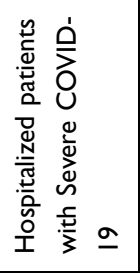 & 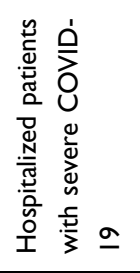 & 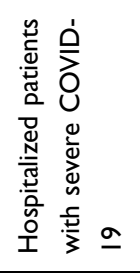 & 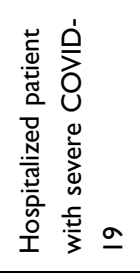 & 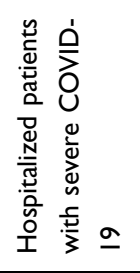 & 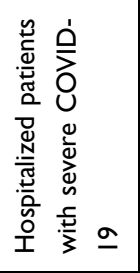 & 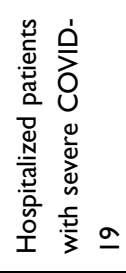 \\
\hline 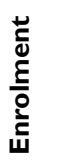 & $\stackrel{\leftrightarrow}{+}$ & $\bar{N}$ & $\underline{\underline{n}}$ & $\simeq$ & $\stackrel{2}{2}$ & $\cong$ & $\stackrel{\circ}{\circ}$ & น & $\bar{n}$ & $\bar{n}$ \\
\hline $\bar{n} z$ & & & & & & & & & & \\
\hline
\end{tabular}




\begin{tabular}{|c|c|c|c|c|c|}
\hline 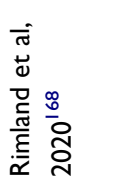 & 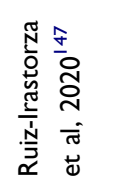 & 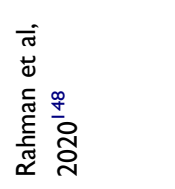 & 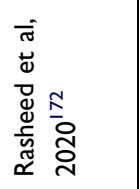 & 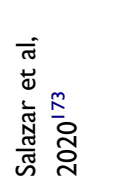 & 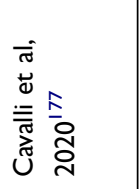 \\
\hline 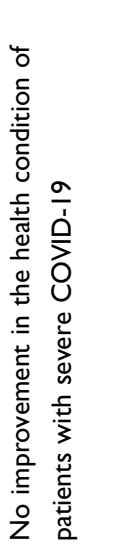 & 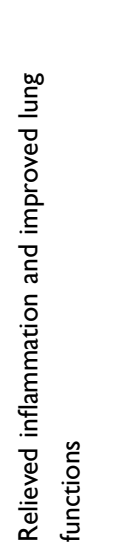 & 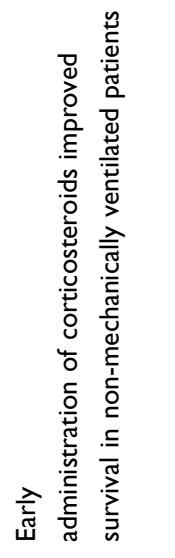 & 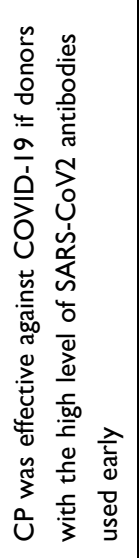 & 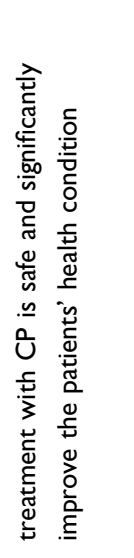 & 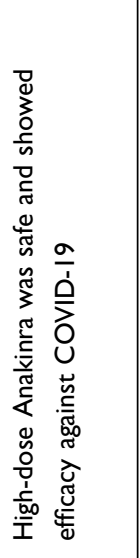 \\
\hline 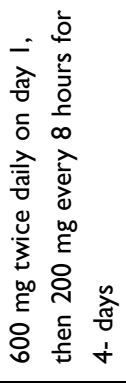 & 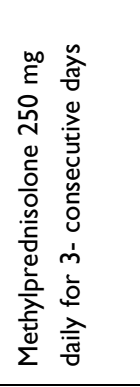 & & 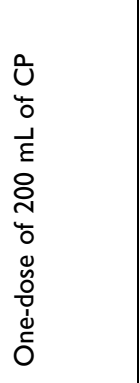 & 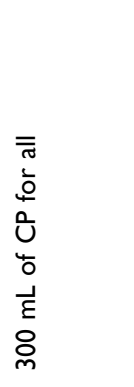 & 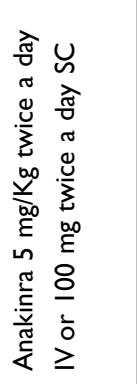 \\
\hline 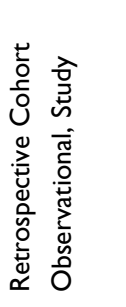 & 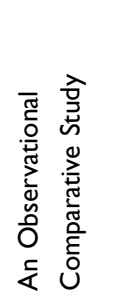 & 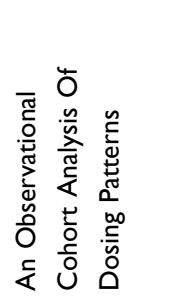 & 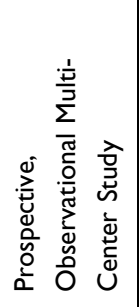 & 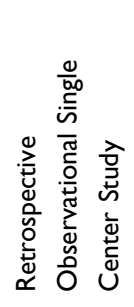 & 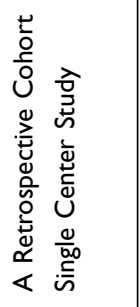 \\
\hline$\underset{r}{N}$ & 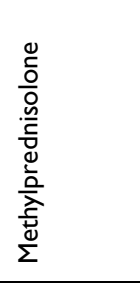 & 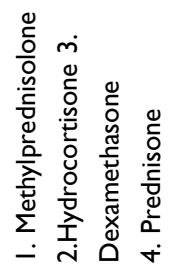 & 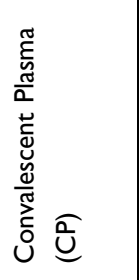 & 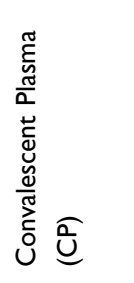 & 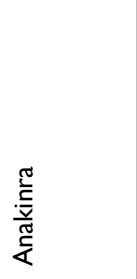 \\
\hline 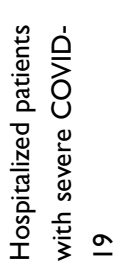 & 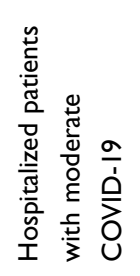 & 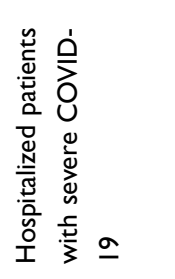 & 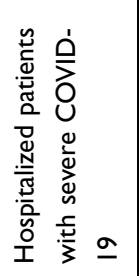 & 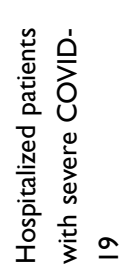 & 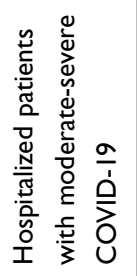 \\
\hline$=$ & $\stackrel{\stackrel{d}{\sim}}{ }$ & $\stackrel{m}{\underline{m}}$ & $q$ & $\stackrel{\sim}{\sim}$ & in \\
\hline
\end{tabular}


did not appear to yield significant negative test results among 150 patients with mild to moderate COVID-19 versus standard care. ${ }^{107}$ Another study involving 33 patients in an RCT and 37 in a retrospective study also failed to demonstrate that HCQ shortened viral shedding. ${ }^{108}$ Molina et al (2020), in their initial study in France, also found no strong evidence of antiviral activity by HCQ. ${ }^{109}$

In contrast, an initial trial in China involving 48 patients with moderate COVID-19 found that chloroquine (CQ) or HCQ showed a trend towards a decrease in the duration of hospital stay and lung computerized RCT. ${ }^{110}$ Andrew et al (2020) also reported that HCQ significantly reduced the duration of hospital stay in a retrospective observational study. ${ }^{111}$ Guérin et al (2020), in their initial study in France involving 88 patients, also found that AZM plus HCQ favorably improved the patient's health status. ${ }^{112}$ Lagier et al (2020) found that HCQ combined with AZM for at least 3 days decreased the need for Intensive Care Unit (ICU) among 3737 COVID-19 patients in Marseille, France. ${ }^{113}$ Arshad et al (2020) also showed that among 2541 patients treated with HCQ alone, AZM alone, and HCQ plus AZM, treatment with HCQ or HCQ plus AZM was associated with lower mortality. ${ }^{114}$ Another study from China indicated that treatment with HCQ alone reduced the incidence of pneumonia among 62 patients with mild COVID-19 in a randomized clinical trial. ${ }^{115}$ Million et al (2020) also found that the combination of HCQ with AZM reduced mortality in patients with uncomplicated COVID19. ${ }^{116}$ Gautret et al (2020) also showed that AZM combined with HCQ significantly reduced the multiplication of the COVID-19 virus. ${ }^{117}$ The initial study by Gautret et al (2020) demonstrated the potential benefits of HCQ; however, as mentioned, the study was severely criticized as no control arm was involved. ${ }^{30,39,40}$

Overall, though, HCQ was found not to effectively prevent or treat mild, moderate, or severe COVID-19 infections in prospective randomized clinical trials and retrospective and observational studies. This, together with safety concerns, prompted the WHO to drop HCQ from the solidarity trial. ${ }^{44}$ The National Institute of Health in the USA also dropped HCQ from its studies because HCQ could not slow disease progression, pneumonia, acute respiratory distress, and death. ${ }^{118}$

\section{Lopinavir (LPV)/Ritonavir (r)}

Lopinavir/ritonavir blocks an enzyme known as 3CLpro, a major $\mathrm{CoV}$ protease enzyme that cleaves the polyproteins during viral replication by arresting COVID-19 multiplication and spread. Consequently, an $\mathrm{LPV} / \mathrm{r}$ combination may be useful in treating mild, moderate, and severe COVID-19 infection. ${ }^{119}$ In a triple therapy study, LPV/r was potentially promising by suppressing the viral load, while methylprednisolone reduced the incidence of pneumonia and the need for ICU admission. ${ }^{120}$ Ye et al (2020), in an early study in China involving 47 patients, revealed that lopinavir/ritonavir lowered body temperature and restored normal physiological functions more effectively than seen in a control group. ${ }^{121}$ A retrospective observational study was undertaken in Nigeria by Bowale et al (2020), who reported the benefit of LPV/r as it significantly reduced hospital stay duration. ${ }^{122}$ However, in an open-label, randomized controlled clinical trial, LPV/r produced no clinical evidence of efficacy among 199 COVID-19 patients. ${ }^{123} \mathrm{Li}$ et al (2020), in their randomized controlled study in China involving 86 patients, also found no clinical benefit from $\mathrm{LPV} / \mathrm{r}$ versus umifenovir among patients with mild to moderate COVID-19. ${ }^{124}$ Also, the UK recovery study randomizing 1596 patients to $\mathrm{LPV} / \mathrm{r}$ versus 3376 patients receiving usual care showed no benefit from LPV/r. ${ }^{51}$ Interestingly, recent studies revealed that the addition of another drug like methylprednisolone or umifenovir to LPV/r improves the treatment outcomes. ${ }^{120,125}$ However, another study, despite the addition of umifenovir, reported otherwise. ${ }^{126}$ Equally, lopinavir, in addition to oseltamivir and ganciclovir, showed no efficacy in all groups of COVID-19 patients enrolled. ${ }^{127}$ Consequently, further studies are needed before any recommendation can be made regarding LPV/r's role in managing patients with COVID19. In the meantime, as mentioned, the WHO has suspended LPV/r from the solidarity trial. ${ }^{44}$

\section{Favipiravir (FPV)}

The mechanism of action of favipiravir involves inhibition of RNA-dependent RNA polymerase enzyme, thereby inhibiting viral RNA synthesis. ${ }^{128}$ Chen et al (2020) undertook a recent clinical trial involving FPV combined with umifenovir. The authors reported that umifenovir and FPV were not effective in treating patients with COVID19 infection among 240 patients enrolled. ${ }^{128}$ Similarly, another study indicated that FPV was not effective in reducing viral load. ${ }^{129}$ However, two recent clinical trials revealed that FPV administered to COVID-19 patients showed a faster recovery than the control group. ${ }^{130,131}$ Overall, there is currently insufficient evidence to suggest 
that the FPV has significant antiviral activity against COVID-19 and could be recommended as a future treatment.

\section{Triazavirin}

Triazavirin is a guanine nucleotide analog antiviral initially developed in Russia. Triazavirin acts via inhibition of RNA-dependent RNA polymerase enzyme, thereby inhibiting viral RNA synthesis. ${ }^{132}$ In their study, Wu et al (2020) found that patients administered triazavirin required fewer respiratory, cardiac, hepatic, or renal support therapies than the control group. ${ }^{132}$ However, despite the possible benefit observed, a large randomized, controlled clinical trial is needed before the place of triazavirin can be determined in COVID-19 patients.

\section{Umefenovir}

The mechanism of antiviral activity of umefenovir is via blockade of viral entry into the host's cell and therefore protects it from viral infection. ${ }^{133}$ Lian et al (2020), in a retrospective observational study, reported that umifenovir was no better than the control group in suppressing the viral load when administered to 81 COVID-19 patients. ${ }^{133}$ This implied that umifenovir monotherapy should not be used in the management of COVID-19 patients.

\section{Remdesivir}

Remdesivir is a monophosphoramidate adenosine analogue that targets and inhibits RNA-dependent RNA polymerase enzyme, thereby inhibiting viral RNA synthesis. Remdesivir has both in-vitro and in-vivo antiviral activity against several viruses, including SARS-CoV-2. As a result, remdesivir may have a place in patient treatment with mild to moderate COVID-19 disease. ${ }^{119,134}$ Conversely, the initial studies with remdesivir failed to demonstrate clinical benefit over placebo; however, there were concerns that the investigations were underpowered. $^{32,135}$ Spinner et al (2020), in a study involving 596 COVID-19 patients, found those patients randomized to a 10-day course of remdesivir did not show a statistically significant improvement in their health status versus standard care randomized. However, those receiving a 5-day course had improved outcomes versus standard care, but the clinical impact was uncertain. ${ }^{38}$ On the other hand, Grein et al (2020) among 61 patients with severe COVID-19 who took remdesivir for compassionate use showed significant improvement in the health status of $68 \%$ of those enrolled. ${ }^{136}$ More recently, a larger-scale study conducted among 1062 COVID-19 patients by $\mathrm{NIH}$ in the USA showed encouraging results, including a reduction in recovery time and a trend towards lower mortality. ${ }^{137}$ This resulted in an emergency use and authorization by the US Food and Drug Administration and an endorsement by the European Medicines Agency and the National Health Services in the UK. ${ }^{45,138-141}$ Patient's liver and kidney function must be monitored during treatment to help minimize any adverse drug reactions. ${ }^{142}$ However, more recent evidence has resulted in WHO guidelines no longer recommending the use of remdesivir in hospitalized patients with COVID-19. This is based on the reports that remdesivir could not reduce mortality, the need for mechanical ventilation, or the duration of hospital stay. ${ }^{134,143}$ Consequently, further large-scale RCTs are needed to better understand the role of remdesivir in the management of patients with COVID-19. ${ }^{78}$

\section{Dexamethasone and Other Steroids}

The mechanism of action of corticosteroids in the treatment of patients with COVID-19 involves inhibition of inflammatory mediators and the inflammatory process. It begins with pro-inflammatory genes that encode cytokines, chemokines, cell adhesion molecules, inflammatory enzymes, and receptors. ${ }^{134,144}$ Recent WHO guidelines give a strong recommendation on the use of systemic corticosteroids in patients with severe COVID-19; however, it discourages the use of systemic corticosteroids in patients with mild-moderate COVID-19 infection. ${ }^{134}$ This is based on the findings of the UK recovery group lead by Horby et al (2020), where dexamethasone appears to be the most promising treatment to date as it significantly reduced mortality and the duration of hospital stay among 6425 patients with severe COVID-19. ${ }^{36}$ In another study involving Intensive Care Unit (ICU) patients, early administration of dexamethasone also reduced the duration of mechanical ventilation and mortality. ${ }^{145}$ Methylprednisolone has also been shown to significantly decrease lung inflammation among 85 COVID-19 patients. ${ }^{146}$ Ruiz-Irastorza et al (2020) in their study involving 242 patients also showed that methylprednisolone was effective in improving respiration among 242 COVID-19 patients. ${ }^{147}$ Rahman et al (2020) in an observational study involving 136 COVID-19 patients admitted to ICU also found that early administration of steroids improved survival and decreased ICU stay. ${ }^{148}$ In line with this, a recent meta-analysis from the WHO rapid appraisal team reported that critically ill COVID-19 
patients who received systemic corticosteroids were associated with lower mortality than placebo or usual care. ${ }^{35}$ Mikluska et al (2020) also reported that methylprednisolone on its own or combined with tocilizumab among 130 COVID-19 patients improve outcomes in non-intubated patients with COVID-19 pneumonia. ${ }^{27}$

\section{Tocilizumab (TCZ)}

Tocilizumab is a humanized monoclonal antibody that inhibits the interleukin-6 (IL-6) receptor. It is employed in the treatment of rheumatoid arthritis and other autoinflammatory processes. It is also useful in treating severe cytokine release syndrome (CRS) induced by the chimeric antigen receptor. Consequently, TCZ, an IL-6 receptor blocker, may be suitable in treating patients with severe pneumonia. ${ }^{149}$ Toniati et al (2020) found that TCZ significantly reduced the need for mechanical ventilation and improved lung function among 100 COVID-19 patients. ${ }^{150}$ Sciascia et al also found in an off-label, nonrandomized, single-center study that tocilizumab relieved bronchial inflammation among 63 COVID-19 patients. ${ }^{151}$ A recent retrospective, observational study by Biran et al (2020) demonstrated that TCZ significantly reduced mortality among 630 COVID-19 patients requiring ICU. ${ }^{152}$ Guaradi et al (2020) also showed that TCZ effectively relieved inflammation among patients with severe COVID-19. ${ }^{153}$ Moreno et al (2020) also found that TCZ decreased mortality and duration of hospital stay in critically ill patients but seemed to have a high risk of serious infections. ${ }^{154}$ Similar outcomes were reported in another related study involving 158 COVID-19 patients. $^{155}$ Somers et al (2020) also found that tocilizumab significantly decreased mortality among 154 patients with severe COVID-19. ${ }^{156}$ Furthermore, in research carried out by Yale University School of Medicine, tocilizumab reduced the need for mechanical ventilation and improved inflammatory biomarkers. ${ }^{157}$ Other studies also reported comparable outcomes. ${ }^{158,159} \mathrm{Xu}$ et al (2020) from China revealed that tocilizumab significantly improved clinical outcomes and reduced mortality among patients with severe COVID-19. ${ }^{28}$ It also reduced the risk of cytokine storms among COVID-19 patients in another study. ${ }^{160}$ Research undertaken by Klopfenstein et al (2020) also indicated that TCZ reduced the number of patients requiring ICU and death in patients with severe COVID-19. ${ }^{161}$ Similar outcomes were reported by Gupta et al 2020. ${ }^{162}$ The abilities of the TCZ to relive inflammation and cytokine storms among COVID-19 patients were further justified in a recent meta-analysis. ${ }^{163}$

In contrast, Colaneri et al (2020) reported that TCZ did not reduce mortality or the number of ICU admission among 112 patients with severe COVID-19. ${ }^{149}$ Knorr et al (2020), in a study conducted in the US involving 66 patients, also found limited clinical improvement with TCZ in patients with severe COVID-19 in a retrospective observational study. ${ }^{164}$ Kewan et al (2020) also reported similar therapeutic failure in treating COVID-19 patients with TCZ. ${ }^{165}$ Other researchers reported similar findings. ${ }^{165-170}$ In addition to a lack of effectiveness with TCZ, Moreno Perez et al (2020) also found that critically ill patients taking TCZ appeared to have a high risk of severe infections. ${ }^{154}$ Consequently, despite the promise shown by tocilizumab in relieving inflammation, decreased mortality, and duration of hospital stay in some studies, we believe more research is needed before the place of TCZ in the treatment of patients with COVID19 can be fully elucidated. ${ }^{163}$

\section{Other Treatments}

Other investigational treatments for COVID-19 involve convalescent plasma (CP) from previously infected and recovered patients. ${ }^{171}$ Duan et al (2020), administering CP from donors with a high level of SARS-CoV-2 antibodies early in the disease, found this effective in boosting immunity among ten COVID-19 patients enrolled in the clinical trial. ${ }^{172}$ In addition, CP produced faster recovery among 49 COVID-19 patients in a retrospective observational study. ${ }^{173}$ Related findings were obtained among twenty-five COVID-19 patients enrolled in a similar investigation. ${ }^{174} \mathrm{~A}$ recent meta-analysis revealed that previous studies involving the transfusion of $\mathrm{CP}$ to patients with deadly Ebola, SARS-CoV-1, and H1N1 viruses improved patients' immunity, suppressed the viral load, and reduced the duration of hospital stay and mortality with minimal side effects. ${ }^{175}$ Nonetheless, the Indian Council of Medical Research published an openlabel phase-2 multicenter randomized controlled trial (placid trial). Their research findings revealed that $\mathrm{CP}$ did not decrease COVID-19 severity and mortality rate. ${ }^{176}$ Consequently, further studies are needed before the use of CP in patients with COVID-19 can be fully elucidated.

Anakinra is a proinflammatory interleukin (IL)- $1 \alpha$ and IL-1 $\beta$ inhibitor which has been used with some success to treat macrophage activation syndrome (MAS) and severe 
cytokine release syndrome (CRS) caused by various inflammatory conditions. ${ }^{177}$ Cavalli et al (2020) have reported that high-dose anakinra was safe and associated with clinical improvement in patients with moderate COVID-19. ${ }^{178}$ However, further studies are imperative before any comments can be made regarding the effectiveness and safety of anakinra in patients with COVID-19.

\section{Discussion}

The studies reviewed indicate concerns with a number of the proposed treatments. Principally, HCQ with or without AZM appeared unable to effectively prevent or treat COVID-19 alongside potential harm despite the initial hype. The lack of efficacy of HCQ with or without AZM implies they were not capable of blocking the COVID-19 virus from binding and invading the host's cell. This suggests the possible use of multiple mechanisms of pathogenesis by the COVID-19 virus. Henceforth, an evidence-based approach is critical when authorities and governments recommend treatment modalities. This is endorsed by the subsequent findings with lopinavir/ritonavir and the more recent studies with remdesivir. Lopinavir/ ritonavir and remdesivir were not effective in inhibiting COVID-19 replication within the host's cell. This signifies that the COVID-19 virus may employ multiple replication modes beyond inhibition of protease and RNA polymerase enzymes. There is more robust clinical evidence for dexamethasone and methylprednisone in symptomatic treatment. These steroids significantly reduced inflammation due to viral pneumonia, thereby decreasing mortality, the need for mechanical ventilation, and the duration of hospital stay.

However, further studies are needed to define optimal doses fully. Furthermore, promising results were obtained with TCZ to improve lung function and reduce mortality among patients with severe COVID-19. TCZ targets and inhibits interleukin-6 leading to cytokine storm prevention responsible for respiratory depression and deaths. However, again more studies are needed to assess its role and value given the contradictory findings to date. Convalescent plasma may also have a place in the treatment of mild COVID-19 when administered early. This is because of the timely administration of CP to COVID-19 patients helps develop strong immunity and eliminates the SARS-CoV-2 virus. However, further randomized clinical trials are needed involving many patients before $\mathrm{CP}$ can be fully integrated into conventional treatment.
Consequently, healthcare providers should observe caution in any off-label prescription until confirmatory studies are reported. Despite the earlier reviews undertaken on various treatment options for COVID-19, we further investigated the outcome of several randomized controlled clinical trials and retrospective observational studies. This objective was to build on existing knowledge and to stimulate further debate that could lead to the emergence of a more evidence-based therapeutic option.

\section{Conclusions}

COVID-19 Pandemic has affected almost every country in the world. The disease currently has killed over one million people globally and has significantly ravaged individuals, communities, national and international economies. The United Nations and countries have made several efforts to reduce prevalence and mortality rates. Many medicines, including repurposed medicines, ${ }^{179-181}$ have been the subject of clinical trials given the urgent need to reduce current morbidity and mortality rates. Pharmaceutical companies and reputable health institutions have also been working to produce an effective and safe vaccine. Whilst there was initial hype surrounding medicines such as HCQ, dexamethasone has shown promise in the symptomatic treatment of COVID-19 patients with severe pneumonia.

Nonetheless, to date, no single confirmed therapeutic option for patients with COVID-19 has been fully elucidated. This endorses the need for all key stakeholders to instigate evidenced-based approaches and not rush to administer unproven treatments that may result in more harm than good. We further await the reports of ongoing research, including new vaccines, with the hope of providing clinicians with more information to enable evidence-based decision-making on the treatment options for COVID-19.

\section{Limitations of the Study}

1) Heterogeneous cohort studies were included.

2) Pre-print articles were also included because research in COVID-19 infection is a novel and continuously evolving study area.

3) The research has no supporting fund to enable the purchase of articles that are not open access.

\section{Acknowledgment}

We wish to extend special gratitude to all Department of Pharmacology staff, Faculty of Pharmaceutical Sciences, Bayero University, Kano, Nigeria. 


\section{Author Contributions}

All authors made a significant contribution to the work reported, whether that is in the conception, study design, execution, acquisition of data, analysis and interpretation, or in all these areas; took part in drafting, revising or critically reviewing the article; gave final approval of the version to be published; have agreed on the journal to which the article has been submitted; and agree to be accountable for all aspects of the work.

\section{Funding}

This paper was not funded.

\section{Disclosure}

The authors report no conflicts of interest for this work and declare that they do not have any financial involvement or affiliations with any organization, association, or entity directly or indirectly with the subject matter or materials presented in this article. This also includes honoraria, expert testimony, employment, ownership of stocks or options, patents or grants received or pending, or royalties.

\section{References}

1. Deng SQ, Peng HJ. Characteristics of and public health responses to the coronavirus disease 2019 outbreak in China. J Clin Med. 2020;9(2):575.

2. Chen N, Zhou M, Dong X, et al. Epidemiological and clinical characteristics of 99 cases of 2019 novel coronavirus pneumonia In Wuhan, China: a descriptive study. Lancet. 2020;395 (10223):507-513.

3. Huang C, Wang Y, Li X, et al. Clinical Features of patients infected with 2019 novel coronavirus in Wuhan, China. Lancet. 2020;395(10223):497-506.

4. Wang Y, Wang Y, Chen Y, Unique Epidemiological QQ. Clinical features of the emerging 2019 novel coronavirus pneumonia (covid-19) implicate special control measures. J Med Virol. 2020;92(6):568-576.

5. Adhikari S, Meng S, Wu Y, et al. Epidemiology, causes, clinical manifestation and diagnosis, prevention and control of coronavirus disease (covid-19) during the early outbreak period: a scoping review. Infect Dis Povert. 2020;9(1):29.

6. Guan W-J, Ni Z-Y, Hu Y, et al. Clinical characteristics of coronavirus disease 2019 in China. $N$ Engl J Med. 2020;382(18):1708-1720.

7. Williamson E, Walker A, Bhaskaran K, et al. Factors associated with covid-19-related death using OpenSAFELY. Nature. 2020;584(7821):430-436.

8. Du RH, Liang L, Yang CQ, et al. Predictors Of Mortality For Patients With Covid-19 Pneumonia Caused By Sars-Cov-2: A Prospective Cohort Study. Eur Respir J. 2020;55(5):2000524.

9. Basu S. Non-Communicable Disease Management In Vulnerable Patients During Covid-19. Indian J Med Ethics. 2020;V (2):103-105.

10. Huang I, Lim MA, Pranata R. Diabetes Mellitus Is Associated With Increased Mortality and Severity Of Disease In Covid-19 Pneumonia - a systematic review, Meta-Analysis, and MetaRegression. Diabetol Metab Syndr. 2020;14(4):395-403.
11. Alqahtani J, Oyelade T, Aldhahir AM, et al. Prevalence, Severity, and Mortality Associated With Copd and Smoking In Patients With Covid-19: A Rapid Systematic Review, and Meta-Analysis. PLoS One. 2020;15(5):E0233147.

12. Vardavas CI, Nikitara K. Covid-19, and smoking: a systematic review of the evidence. Tob Induc Dis. 2020;18:20.

13. Pareek N. Preliminary estimates of the prevalence of selected underlying health conditions among patients with coronavirus disease 2019 - United States, february 12-march 28, 2020. MMWR. 2020;69(13):382-386.

14. Garima S, Singh N. Fatality in COVID 19: an overview of causes of death and organ involvement. Int $J$ Adv Med. 2020;7:1190-1193.

15. Khunti K, Singh AK, Pareek M, Hanif W. Is ethnicity linked to incidence or outcomes of Covid-19? BMJ. 2020;369:M1548.

16. Pareek M, Bangash MN, Pareek N, et al. Ethnicity and Covid-19: an urgent public health research priority. Lancet. 2020;395 (10234):1421-1422.

17. World Health Organization. Coronavirus Disease (Covid-19) Situation Report September 20; 2020. Available from: Https:// Www.Who.Int/Docs/Default-Source/Coronaviruse/SituationReports/20200921-Weekly-Epi-Update-6.Pdf?Sfvrsn=D9cf9496_ 6. Accessed December 15, 2020.

18. Cao Y, Li L, Feng Z, et al. Comparative genetic analysis of the novel coronavirus (2019-nCoV/SARS-CoV-2) receptor ACE2 in different populations. Cell Dis. 2020;6:11.

19. Lu R, Zhao X, Li J, et al. Genomic Characterization and Epidemiology Of 2019 Novel Coronavirus: implications For Virus Origins and Receptor Binding. Lancet. 2020;395 (10224):565-574.

20. Fehr AR, Perlman S. Coronaviruses: an Overview Of Their Replication and Pathogenesis. Methods Mol Biol. 2015;1282:1-23.

21. Wang Y, Grunewald M, Perlman S. Coronaviruses: an Updated Overview Of Their Replication and Pathogenesis. Methods Mol Biol. 2020;2203:1-29.

22. Hippisley-Cox J, Young D, Coupland C, et al. Risk of severe COVID-19 disease with ACE inhibitors and angiotensin receptor blockers: cohort study including 8.3 million people. Heart. 2020;106(19):1503-1511.

23. Kurdi A, Abutheraa N, Akil L, Godman B. A systematic review and meta-analysis of the use of renin-angiotensin system drugs and COVID-19 clinical outcomes: what is the evidence so far? Pharmacol Res Perspect. 2020;8(6):e00666. doi:10.1002/ prp2.666

24. World Health Organization. Laboratory Testing For Coronavirus Disease 2019 (Covid-19) In Suspected Human Cases: interim Guidance; 2020. Available from Https://Apps.Who.Int/Iris/ Bitstream/Handle/10665/331329/Who-Covid-19-Laboratory -2020.4-Eng.Pdf.

25. Amrane S, Tissot-Dupont H, Doudier B, et al. Rapid Viral Diagnosis and Ambulatory Management of Suspected Covid-19 Cases Presenting At The Infectious Diseases Referral Hospital In Marseille, France, - January 31 To March 1, 2020: A Respiratory Virus Snapshot. Travel Med Infect Dis. 2020;36:101632.

26. Khan M, Khan H, Khan S, Nawaz M. Epidemiological and Clinical Characteristics Of Coronavirus Disease (Covid-19) Cases At A Screening Clinic During The Early Outbreak Period: A Single-Center Study. J Med Microbiol. 2020;69 (8):1114-1123.

27. Mikulska M, Nicolini LA, Signori A, et al. Tocilizumab and Steroid Treatment In Patients With Covid-19 Pneumonia. PLoS One. 2020;15(8):E0237831.

28. Xu X, Han M, Li T, et al. Effective Treatment Of Severe Covid-19 Patients With Tocilizumab. Proc Natl Acad Sci USA. 2020;117(20):10970-10975. 
29. Ogunleye OO, Basu D, Mueller D, et al. Response To The Novel Corona Virus (Covid-19) Pandemic Across Africa: successes, Challenges, and Implications For The Future. Front Pharmacol. 2020;11:1205.

30. Gautret P, Lagier JC, Parola P, et al. Hydroxychloroquine and Azithromycin As A Treatment Of Covid-19: results Of An Open-Label Non-Randomized Clinical Trial. Int $J$ Antimicrob Agents. 2020;105949.

31. Das S, Bhowmick S, Tiwari S, Sen S. An Updated Systematic Review Of The Therapeutic Role Of Hydroxychloroquine In Coronavirus Disease-19 (Covid-19). Clin Drug Investig. 2020;40(7):591-601

32. Wang Y, Zhang D, Du G, et al. Remdesivir In Adults With Severe Covid-19: A Randomized, Double-Blind, Plsbo-Controlled, Multicentre Trial. Lancet. 2020;395(10236):1569-1578.

33. Siemieniuk RA, Bartoszko JJ, Ge L, et al. Drug Treatments For Covid-19: living Systematic Review and Network Meta-Analysis. BMJ. 2020;370:M2980.

34. Frediansyah A, Nainu F, Dhama K, Mudatsir M, Harapan H. Remdesivir and Its Antiviral Activity Against Covid-19. A Systematic Review. Clin Epidemiol Glo Health. 2020. doi:10.1016/J.Cegh.2020.07.011

35. Sterne JAC, Murthy S, Diaz JV, et al. Association Between Administration Of Systemic Corticosteroids and Mortality Among Critically Ill Patients With Covid-19: A Meta-Analysis. JAMA. 2020. doi:10.1001/Jama.2020.17023

36. Horby P, Lim W, Emberson JR, et al. Dexamethasone In Hospitalized Patients With Covid-19 - Preliminary Report. N Engl J Med. 2020.

37. Ldj B, Brodie D, Calfee CS. Severe Covid-19 Infections-Knowledge Gained and Remaining Questions. JAMA Intern Med. 2020.

38. Spinner C, Gottlieb R, Criner G, et al. Effect of Remdesivir Vs. Standard Care on Clinical Status at 11 Days In Patients With Moderate Covid-19: A Randomized Clinical Trial. JAMA. 2020;324(11):1048-1057.

39. Isac/Elsevier. Joint Isac and Elsevier Statement On Gautret et al. Paper [Pmid 32205204]; 2020. Available from: Https:/Www. Isac.World/News-and-Publications/Isac-Elsevier-Statement.

40. International Society Of Antimicrobial Chemotherapy. Official Statement From International Society Of Antimicrobial Chemotherapy (ISAC) - Hydroxychloroquine and Azithromycin as a Treatment Of Covid-19: results Of An Open-Label NonRandomized Clinical Trial (Gautret P et al. Pmid 32205204). Available from: Https:/Www.Isac.World/News-and-Publications /Official-Isac-Statement.

41. Borba MGS, Almeida Val FF, Sampaio VS, et al. Chloroquine Diphosphate In Two Different Dosages As Adjunctive Therapy Of Hospitalized Patients With Severe Respiratory Syndrome In The Context Of Coronavirus (Sars-Cov-2) Infection: preliminary Safety Results Of A Randomized, Double-Blinded, Phase IIb Clinical Trial (Clorocovid-19 Study). Medrxiv Preprint. 2020.

42. Abena P, Decloedt E, Bottieau E, et al. Chloroquine and Hydroxychloroquine For The Prevention Or Treatment Of Covid-19 In Africa: caution For Inappropriate Off-Label Use In Healthcare Settings. Am J Trop Med Hyg. 2020;102 (6):1184-1188

43. Godman B. Combating Covid-19: lessons Learnt, Particularly Among Developing Countries and The Implications. Bangladesh J Med Sci. 2020;S103-8.

44. World Health Organization. WHO Discontinues Hydroxychloroquine and Lopinavir/Ritonavir Treatment Arms For Covid-19; 2020. Available from: Https:/Www.Who.Int/ News-Room/Detail/04-07-2020-Who-DiscontinuesHydroxychloroquine-and-Lopinavir-Ritonavir-Treatment-ArmsFor-Covid-19. Accessed December 15, 2020.
45. BMJ Best Practice Coronavirus Disease 2019 (Covid-19); 2020 Available from: https://bestpractice.bmj.com/topics/en-gb /3000168/pdf/3000168/coronavirus\%20disease $\% 202019 \% 20 \%$ 28covid-19\%29.pdf. Accessed December 15, 2020.

46. Littlejohn E. Hydroxychloroquine Use In The Covid-19 Patient. Clevel Clin J Med. 2020.

47. ECDC. Vaccines and Treatment Of Covid-19; 2020. Available from: Https:/Www.Ecdc.Europa.Eu/En/Covid-19/LatestEvidence/Vaccines-And-Treatment.

48. Chorin E, Wadhwani L, Magnani S, et al. QT Interval Prolongation and Torsade De Pointes In Patients With Covid-19 Treated With Hydroxychloroquine/Azithromycin. Heart Rhythm. 2020;17(9):1425-1433. doi:10.1016/J.Hrthm.2020.05.014

49. Gevers S, Msg K, Wijnans E, Van Nieuwkoop C. Safety Considerations For Chloroquine and Hydroxychloroquine In The Treatment Of Covid-19. Clin Microbiol Infect. 2020;26 (9):1276-1277. doi:10.1016/J.Cmi.2020.05.006

50. The US. FDA Cautions Against Use Of Hydroxychloroquine Or Chloroquine For Covid-19 Outside Of The Hospital Setting Or A Clinical Trial Due To Risk Of Heart Rhythm Problems. 2020 Available from Https://Www.Fda.Gov/Drugs/Drug-Safety-AndAvailability/Fda-Cautions-Against-Use-Hydroxychloroquine-OrChloroquine-Covid-19-Outside-Hospital-Setting-Or. Accessed October 7, 2020.

51. Recovery Trial. Statement From The Chief Investigators Of The Randomised Evaluation Of Covid-19 Therapy (Recovery) Trial On Lopinavir-Ritonavir, 2020. No Clinical Benefit From Use Of Lopinavir-Ritonavir In Hospitalized Covid-19 Patients Studied In Recovery; 2020. Available from: Https://Www.Recoverytrial.Net/ Files/Lopinavir-Ritonavir-Recovery-Statement-29062020_Final.Pdf.

52. Wang C. Response to Covid-19 In Taiwan: big Data Analytics, New Technology, and Proactive Testing. JAMA. 2020;323 (14):1341-1342. doi:10.1001/Jama.2020.3151

53. Khd T. Movement Control as An Effective Measure Against Covid-19 Spread in Malaysia: an Overview. Z Gesundh Wiss. 2020;1-4. doi:10.1007/S10389-020-01316-W

54. Hoang VM, Hoang H, Khuong Q. Describing the Pattern of The Covid-19 Epidemic in Vietnam. Glob Health Action. 2020;13 (1):1776526. doi:10.1080/16549716.2020.1776526

55. Courtemanche C, Garuccio J, Le A, Pinkston J, Yelowitz A. Strong Social Distancing Measures in The United States Reduced the Covid-19 Growth Rate. Health Aff. 2020;39 (7):1237-1246. doi:10.1377/Hlthaff.2020.00608

56. Ng Y, Li Z, Chua Y, et al. Evaluation of The Effectiveness of Surveillance and Containment Measures for The First 100 Patients with Covid-19 In Singapore - January 2-February 29, 2020. MMWR Morb Mortal Wkly Rep. 2020;69(11):307-311. doi:10.15585/Mmwr.Mm6911e1

57. Hhp K, Wickramasinghe K, Rippin H, et al. Control of Non-Communicable Diseases in The Covid-19 Response. Lancet. 2020;395(10238):1678-1680. doi:10.1016/S01406736(20)31067-9

58. Abbas K, Van Zandvoort K, Clark A, et al. Lshtm Cmmid Covid-19 Working Group. Routine Childhood Immunization During The Covid-19 Pandemic In Africa: A Benefit-Risk Analysis Of Health Benefits Versus Excess Risk Of Sars-Cov-2 Infection. Lancet Glob Health. 2020;8(10):E1264-E1272. doi:10.1016/S2214-109x(20)30308-9

59. Afriyie D, Asare G, Amponsah S, Godman B. Covid-19 Pandemic In Resource-Poor Countries: challenges, Experiences, and Opportunities In Ghana. J Infect Dev Ctries. 2020;14 (8):838-843.

60. Bedford J, Enria D, Giesecke J, et al. Covid-19: towards Controlling Of A Pandemic. Lancet. 2020;395 (10229):1015-1018. 
61. Chu DK, Akl E, Duda S, Solo K, Yaacoub S, Schünemann H. Physical Distancing, Face Masks, and Eye Protection To Prevent Person-To-Person Transmission Of Sars-Cov-2 and Covid-19: A Systematic Review and Meta-Analysis. Lancet. 2020;395 (10242):1973-1987.

62. Nussbaumer-Streit B, Mayr V, Dobrescu A, et al. Quarantine Alone Or In Combination With Other Public Health Measures To Control Covid-19: A Rapid Review. Cochrane Db Syst Rev. 2020;4:Cd013574.

63. Gardaworld. Malaysia: government Closes Borders To All Foreigners Over Covid-19 Concerns March 18/Update 5; 2020. Available from: Https://Www.Garda.Com/Crisis24/News-Alerts /323221/Malaysia-Government-Closes-Borders-To-All-

Foreigners-Over-Covid-19-Concerns-March-18-Update-5. Accessed December 15, 2020.

64. Gardaworld. Malaysia: prime Minister Extends Movement Control Order To April 14/Update 8; 2020. Available from: Https://Www.Garda.Com/Crisis24/News-Alerts/326221/ Malaysia-Prime-Minister-Extends-Movement-Control-Order-ToApril-14-Update-8. Accessed December 15, 2020.

65. Exemplars In Global Health. Emerging Covid-19 Success Story: south Korea Learned The Lessons Of Mers; 2020. Available from: Https://Ourworldindata.Org/Covid-Exemplar-South-Korea. Accessed December 15, 2020.

66. Kim J-H, J A-r A, Min P-K, Bitton A, Gawande A. How South Korea Responded To The Covid-19 Outbreak In Daegu. $N$ Engl J Med Catalyst. 2020;1(4):1-14. doi:10.1056/Cat.20.0159

67. Pham Q, Rabaa M, Duong H, et al. The First 100 Days Of Sars-Cov-2 Control In Vietnam. Clin Infect Dis. 2020.

68. Thwaites G, Rabaa M, Choisy M, Van Doorn R, Luong D, Emerging Covid-19 Success Story: vietnam's Commitment To Containment; 2020. Available from: Https://Ourworldindata.Org/ Covid-Exemplar-Vietnam. Accessed December 15, 2020.

69. Ha BTT, Ngoc Quang L, Mirzoev T, Tai N, Thai P, Dinh P. Combating The Covid-19 Epidemic: experiences From Vietnam. Int J Environ Res Pub Health. 2020;17(9):3125.

70. Tu YF, Chien CS, Yarmishyn AA, et al. A Review Of Sars-Cov-2 and The Ongoing Clinical Trials. Int $J$ Molec Sci. 2020;21 (7):2657.

71. Chowdhury M, Rathod J, Gernsheimer J, Rapid Systematic A. Review Of Clinical Trials Utilizing Chloroquine and Hydroxychloroquine As A Treatment For Covid-19. Acad Emerg Med. 2020;27(6):493-504.

72. Cortegiani A, Ingoglia G, Ippolito M, Giarratano A, Einav S. A Systematic Review On The Efficacy and Safety Of Chloroquine For The Treatment Of Covid-19. J Critic Care. 2020;57:279-283.

73. Ford N, Vitoria M, Rangaraj A, Norris S, Calmy A, Doherty M. Systematic Review Of The Efficacy and Safety Of Antiretroviral Drugs Against Sars, Mers, Or Covid-19: initial Assessment. $J$ Int AIDS Soc. 2020;23(4):E25489.

74. Rajendran DK, Rajagopal V, Alagumanian S, Santhosh Kumar T, Sp SP, Kasilingam D. Systematic Literature Review On Novel Coronavirus Sars-Cov-2: A Threat To Human Era. Virus Disease. 2020;31(2):161-173.

75. Singh A, Singh A, Singh R, Remdesivir MA. In Covid-19: A Critical Review Of Pharmacology, Pre-Clinical and Clinical Studies. Diabeol Metab Syndr. 2020;14(4):641-648.

76. Zhong H, Wang Y, Zhang Z, et al. Efficacy and Safety Of Current Therapeutic Options For Covid-19 - Lessons To Be Learnt From Sars and Mers Epidemic: A Systematic Review and Meta-Analysis. Pharmacol Res. 2020;157:104872.

77. Singh A, Singh A, Singh R, Misra A. Hydroxychloroquine In Patients With Covid-19: A Systematic Review and Meta-Analysis.. Diabeol Metab Syndr. 2020;14(4):589-596.
78. Mccreary E, Angus D. Efficacy Of Remdesivir In Covid-19. JAMA. 2020;324(11):1041-1042.

79. Pastick K, Okafor E, Wang F, et al. Review: hydroxychloroquine and Chloroquine For Treatment Of Sars-Cov-2 (Covid-19). Open Forum Infect Dis. 2020;7(4):Ofaa130.

80. Lam S, Lombardi A, Ouanounou A. Covid-19: A Review Of The Proposed Pharmacological Treatments. Eur $J$ Pharmacol. 2020;886:173451.

81. Khuroo MS. Chloroquine and Hydroxychloroquine In Coronavirus Disease 2019 (Covid-19). Facts, Fiction, and The Hype: A Critical Appraisal. Int J Antimicrob Agents. 2020;56(3):106101.

82. Pathak DSK, Daa S, Thivari DP, Pandey A, Nandy DK. Harish V K Ratna D, et al. No Benefit Of Hydroxychloroquine In Covid19: results Of Systematic Review and Meta-Analysis Of Randomized Controlled Trials". Diabetol Metab Syndr. 2020;14(6):1673-1680.

83. Shah S, Das S, Jain A, Misra D, Negi V. A Systematic Review Of The Prophylactic Role Of Chloroquine and Hydroxychloroquine In Coronavirus Disease-19 (Covid-19). Int J Rheum Dis. 2020;23 (5):613-619.

84. Liu W, Zhou P, Chen K, et al. Efficacy and Safety Of Antiviral Treatment For Covid-19 From Evidence In Studies Of Sars-Cov-2 and Other Acute Viral Infections: A Systematic Review and Meta-Analysis. CMAJ. Canadian Med Assoc J. 2020;192(27): E734-E44.

85. Moher D, Shamseer L, Clarke M, et al. Preferred Reporting Items For Systematic Review and Meta-Analysis Protocols (Prisma-P) 2015 Statement. Syst Rev. 2015;4(1):1.

86. Phrf A, Tbc S, De Assis Acurcio F, et al. Of Life Of Patients With Type 1 Diabetes Mellitus Using Insulin Analog Glargine Compared With Nph Insulin: A Systematic Review and Policy Implications. Patient. 2018;11(4):377-389. doi:10.1007/S40271017-0291-3

87. Manoukian S, Stewart S, Dancer S, et al. Estimating Excess Length Of Stay Due To Healthcare-Associated Infections: A Systematic Review and Meta-Analysis Of Statistical Methodology. J Hosp Infect. 2018;100(2):222-235. doi:10.1016/ J.Jhin.2018.06.003

88. Silva TBC, Phrf A, Araújo V, et al. Safety Of Insulin Glargine Versus Detemir Analysis In Patients With Type 1 Diabetes: systematic Review and Meta-Analysis. Ther Adv Endocrinol Metab. 2018;9(8):241-254. doi:10.1177/2042018818781414

89. Caires De Souza A. Insulin Glargine In A Brazilian State: should The Government Disinvest? An Assessment Based On A Systematic Review. Appl Health Econ Health Policy. 2014;12 (1):19-32. doi:10.1007/S40258-013-0073-6

90. McGregor SK. Energetics Based Modeling of Hydroxychloroquine and Azithromycin Binding to the SARS-CoV-2 Spike (S)Protein-ACE2 Complex. ChemRxiv. 2020. doi:10.26434/chemrxiv. 12015792

91. Boulware D, Pullen M, Bangdiwala A, et al. A Randomized Trial Of Hydroxychloroquine As Postexposure Prophylaxis For Covid-19. N Engl J Med. 2020;383(6):517-525.

92. Chen J, Liu D, Liu L, et al. [A Pilot Study Of Hydroxychloroquine In Treatment of Patients With Moderate Covid-19]. J Zhejiang Univ Med Sci. 2020;49(2):215-219.

93. Skipper C, Pastick K, Engen N, et al. Hydroxychloroquine In Nonhospitalized Adults With Early Covid-19: A Randomized Trial. Ann Int Med. 2020.

94. Mahévas M, Tran V, Roumier M, et al. Clinical Efficacy Of Hydroxychloroquine In Patients With Covid-19 Pneumonia Who Require Oxygen: observational Comparative Study Using Routine Care Data. BMJ. 2020;369:M1844.

95. Mitjà $\mathrm{O}$, Corbacho-Monné $\mathrm{M}$, Ubals $\mathrm{M}$, et al. Hydroxychloroquine For Early Treatment Of Adults With Mild Covid-19: A Randomized-Controlled Trial. Clin Infec Dis. 2020: Ciaa1009. doi:10.1093/Cid/Ciaa1009 
96. Magagnoli J, Narendran S, Pereira F, et al. Outcomes Of Hydroxychloroquine Usage In United States Veterans Hospitalized With Covid-19. Med (NY). 2020.

97. Cavalcanti A, Zampieri F, Rosa R, et al. Hydroxychloroquine With Or Without Azithromycin In Mild-To-Moderate Covid-19. N Engl J Med. 2020;24. doi:10.1056/Nejmoa2019014

98. Recovery Trial. No Clinical Benefit From Use Of Hydroxychloroquine In Hospitalized Patients With Covid-19. 2020. Available from: Https://Www.Recoverytrial.Net/News/StatementFrom-The-Chief-Investigators-Of-The-Randomised-Evaluation-OfCovid-19-Therapy-Recovery-Trial-On-Hydroxychloroquine-5-June2020-No-Clinical-Benefit-From-Use-Of-Hydroxychloroquine-InHospitalised-Patients-With-Covid-19.

99. Geleris J, Sun Y, Platt J, et al. Observational Study Of Hydroxychloroquine In Hospitalized Patients With Covid-19. $N$ Engl J Med. 2020;382(25):2411-2418.

100. Singh S, Khan A, Chowdhry M, Chatterjee A. Outcomes Of Hydroxychloroquine Treatment Among Hospitalized Covid-19 Patients In The United States-Real-World Evidence From A Federated Electronic Medical Record Network. Medrxiv. 2020.

101. Ip A, Da B, Hansen E, et al. Hydroxychloroquine and Tocilizumab Therapy In Covid-19 Patients-An Observational Study. PLoS One. 2020;15(8):E0237693.

102. Ferner R, Aronson J. Chloroquine and Hydroxychloroquine In Covid-19. BMJ. 2020;369:M1432.

103. Nga L, Phuong L, Anh P Hanoi Man Od's On Rumored Malaria Drug Cure For Covid-19, 2020. Available from: Https://E. Vnexpress.Net/News/News/Hanoi-Man-Od-S-On-RumoredMalaria-Drug-Cure-For-Covid-19-4073488.Html.

104. Garcia P, Revet A, Yrondi A, Rousseau V, Degboe Y, Psychiatric Disorders MF. Hydroxychloroquine for Coronavirus Disease 2019 (COVID-19): A VigiBase Study. Drug Safety. 2020;19:1-8.

105. Emmanuel S, Ostlundh L. Psychiatric adverse events with hydroxychloroquine during COVID-19 Pandemic. Asian J Psych. 2020;54(2020):102203.

106. Rhm F, Berwanger O, Fonseca H, et al. Azithromycin in addition to standard of care versus standard of care alone in the treatment of patients admitted to the hospital with severe covid-19 in brazil (coalition ii): a randomized clinical trial. Lancet. 2020;S01406736 .

107. Tang W, Cao Z, Han M, et al. Hydroxychloroquine in patients with mainly mild to moderate coronavirus disease 2019: open-label, randomized controlled trial. BMJ. 2020;369:M1849M.

108. Chen C. A Multicenter, randomized, open-label, controlled trial to evaluate the efficacy and tolerability of hydroxychloroquine and a retrospective study in adult patients with mild to moderate coronavirus disease 2019 (Covid-19). Medrxiv. 2020 doi:10.1101/2020.07.08.20148841

109. Molina J, Delaugerre C, Le Goff J, et al. No evidence of rapid antiviral clearance or clinical benefit with the combination of hydroxychloroquine and azithromycin in patients with severe covid-19 infection. Med Malad Infect. 2020;50(4):384.

110. Chen L, Zhang Z. Efficacy and safety of chloroquine or hydroxychloroquine in moderate type of covid-19: a prospective open-label randomized controlled study. Medrxiv. 2020. doi:10.1101/2020.06.19.20136093

111. Andrew I, Ahn J, Zhou Y, et al. Hydroxychloroquine in the treatment of outpatients with mildly symptomatic covid-19: a multicenter observational study. Medrxiv Infect Dis. 2020. doi:10.1101/2020.08.20.20178772

112. Guérin V, Lévy P, Thomas J-L, et al. Azithromycin and Hydroxychloroquine Accelerate Recovery of Outpatients with Mild/Moderate Covid-19. Asian J Med Health. 2020;18 (7):45-555. doi:10.20944/Preprints202005
113. Lagier JC, Million M, Gautret P, et al. Outcomes Of 3737 Covid-19 Patients Treated With Hydroxychloroquine/Azithromycin and Other Regimens In Marseille, France: A Retrospective Analysis. Travel Med Infect Dis. 2020;36:101791. doi:10.1016/J.Tmaid.2020.101791

114. Arshad S, Kilgore P, Chaudhry Z, et al. Treatment With Hydroxychloroquine, Azithromycin, and Combination In Patients Hospitalized With Covid-19. Intern J Infect Dis. 2020;97:396-403.

115. Chen Z, Hu J, Zhang Z, et al. Efficacy of Hydroxychloroquine in Patients with Covid-19: results of A Randomized Clinical Trial. Medrxiv. 2020. doi:10.1101/2020.03.22.20040758

116. Million M, Lagier JC, Gautret P, et al. Early Treatment of Covid-19 Patients with Hydroxychloroquine and Azithromycin: A Retrospective Analysis of 1061 Cases in Marseille, France. Travel Med Infect Dis. 2020;35:101738. doi:10.1016/J. Tmaid.2020.101738

117. Gautret P, Lagier JC, Parola P, et al. Clinical and Microbiological Effect of a Combination of Hydroxychloroquine and Azithromycin in 80 Covid-19 Patients with at Least A Six-Day Follow Up: A Pilot Observational Study. Travel Med Infect Dis. 2020;34:101663. doi:10.1016/J.Tmaid.2020.101663

118. Nih. Nih Halts Clinical Trial Of Hydroxychloroquine. 2020. Available from: Https:/Www.Nhlbi.Nih.Gov/News/2020/NihHalts-Clinical-Trial-Hydroxychloroquine. Accessed December $15,2020$.

119. Uzunova K, Filipova E, Pavlova V, Vekov T. Insights into antiviral mechanisms of remdesivir, lopinavir/ ritonavir, and chloroquine/hydroxychloroquine affecting the new SARS-CoV-2. Biomed Pharmacother. 2020;131(2020):110668.

120. Hung I, Lung K, Tso E, et al. Triple Combination Of Interferon Beta-1b, Lopinavir-Ritonavir, and Ribavirin In The Treatment Of Patients Admitted To Hospital With Covid-19: an Open-Label, Randomized, Phase 2 Trial. Lancet. 2020;395(10238):1695-1704.

121. Ye X, Luo Y, Xia S, et al. Clinical Efficacy Of Lopinavir/ Ritonavir In The Treatment Of Coronavirus Disease 2019. Eur Rev Med Pharmacol Sci. 2020;24(6):3390-3396.

122. Bowale A, Abayomi A, Idris J, et al. Clinical Presentation, Case Management and Outcomes For The First 32 Covid-19 Patients In Nigeria. The Pan Afr Med J. 2020;35(Supp2):24. doi:10.11604/ Pamj.Supp.2020.35.2.23262

123. Cao B, Wang Y, Wen D, et al. A Trial Of Lopinavir-Ritonavir In Adults Hospitalized With Severe Covid-19. $N$ Engl J Med. 2020;382(19):1787-1799.

124. Li Y, Xie Z, Lin W, et al. Efficacy and Safety Of Lopinavir/ Ritonavir Or Arbidol In Adult Patients With Mild/Moderate Covid-19: an Exploratory Randomized Controlled Trial. Med (NY). 2020. doi:10.1016/J.Medj.2020.04.001

125. Deng L, Li C, Zeng Q, et al. Arbidol Combined With Lpv/R Versus Lpv/R Alone Against Corona Virus Disease 2019: A Retrospective Cohort Study. J Infect. 2020;81(1):E1-E5.

126. Wen $\mathrm{C}, \mathrm{Xie} \mathrm{Z}, \mathrm{Li} \mathrm{Y}$, et al. [Real-world efficacy and safety of lopinavir/ritonavir and arbidol in treating with covid-19: an observational cohort study]. Chinese J Intern Med. 2020;59:E012.

127. Yang X, Yu Y, Xu J, et al. Clinical Course and Outcomes of Critically Ill Patients with Sars-Cov-2 Pneumonia in Wuhan, China: A Single-Centered, Retrospective, Observational Study. Lancet Respir Med. 2020;8(5):475-481. doi:10.1016/S22132600(20)30079-5

128. Chen C, Huang J, Cheng Z, et al. Favipiravir Versus Arbidol for Covid-19: A Randomized Clinical Trial. Medrxiv. 2020. doi:10.1101/2020.03.17.20037432.T

129. Doi Y, Hibino M, Hase R. Randomized, Open-Label Trial of Early versus Late Favipiravir Therapy in Hospitalized Patients with COVID-19. Antimicrob Agents Chemother. 2020;64(12): e01897-1820. 
130. Ivashchenko AA, Dmitriev KA, Vostokova NV, et al. AVIFAVIR for treatment of patients with moderate COVID-19: interim results of a phase II/III multicenter randomized clinical trial. medRxiv. 2020.

131. Cai Q, Yang M, Liu D, et al. Experimental Treatment with Favipiravir For Covid-19: An Open-Label Control Study. [Published Online Ahead of Print, 2020 March 18]. Engineering. 2020.

132. Wu X, Yu K, Wang Y, et al. Efficacy and Safety of Triazavirin Therapy for Coronavirus Disease 2019: A Pilot Randomized Controlled Trial. Engineering. 2020. doi:10.1016/J. Eng.2020.08.011

133. Lian N, Xie H, Lin S, Huang J, Zhao J, Lin Q. Umifenovir Treatment Is Not Associated with Improved Outcomes in Patients with Coronavirus Disease 2019: A Retrospective Study. Clin Microbiology Infect. 2020;26(2020):917e921.

134. Lamontagne F, Agoritsas T, Macdonald H, et al. A living WHO guideline on drugs for covid-19. BMJ. 2020;370:m3379.

135. The Guardian. 'High Hopes' Drug For Covid-19 Treatment Failed In Full Trial; 2020. Available from: Https:/Www.Msn.Com/En$\mathrm{Gb} /$ News/Coronavirus/High-Hopes-Drug-For-Covid-19Treatment-Failed-In-Full-Trial/Ar-Bb136z6g?Ocid=Spartandhp.

136. Grein J, Ohmagari N, Shin D, et al. Compassionate Use Of Remdesivir For Patients With Severe Covid-19. $N$ Engl J Med. 2020;382(24):2327-2336.

137. Beigel JH, Tomashek KM, Le D, et al. Remdesivir For The Treatment Of Covid-19 - Preliminary Report. $N$ Engl $J$ Med. 2020. doi:10.1056/Nejmoa2007764

138. US Food and Drug Administration. Emergency Use Authorization (Eua) For Emergency Use Of Remdesivir For The Treatment Of Hospitalized Coronavirus Disease (Covid-19) Patients; 2020. Available: Https://Www.Fda.Gov/Media/137564/Download. Accessed December 15, 2020.

139. UK Medicines \& Healthcare Products Regulatory Agency. Eams 11972/0001 Remdesivir $100 \mathrm{Mg}$ Powder For Concentrate For Solution For Infusion; 2020. Available: Https://Www.Gov.Uk/ Government/Publications/Early-Access-To-Medicines-SchemeEams-Scientific-Opinion-Remdesivir-In-The-Treatment-OfPatients-Hospitalised-With-Suspected-Or-Laboratory-Confirme /Treatment-Protocol-For-Healthcare-Professionals_Eams -119720001-Remdesivir-100-Mg-Powder-For-Concentrate-ForSolution-For-Infusion.

140. EMA. First Covid-19 Treatment Recommended For Eu Authorization; 2020. Available from: Https:/Www.Ema.Europa. Eu/En/News/First-Covid-19-Treatment-Recommended-EuAuthorisation.

141. Dolin R, Hirsch MS. Remdesivir - An Important First Step. $N$ Engl $J$ Med. 2020;383(19):1886-1887. doi:10.1056/ NEJMe2018715

142. Charan J, Kaur RJ, Bhardwaj P, et al. Rapid review of suspected adverse drug events due to remdesivir in the WHO database; findings and implications. Expert Rev Clin Pharmacol. 2020. doi:10.1080/17512433.2021.1856655

143. Dyer O. Covid-19: remdesivir has little or no impact on survival, WHO trial shows. BMJ. 2020;371:m4057. doi:10.1136/bmj. $\mathrm{m} 4057$

144. Sterne JA, Murthy S, Diaz JV, et al. Association between administration of systemic corticosteroids and mortality among critically ill patients with COVID-19: a meta-analysis. JAMA. 2020;324(13):1330-1341.

145. Villar J, Ferrando C, Martínez D, et al. Treatment for The Acute Respiratory Distress Syndrome: A Multicentre, Randomised Controlled Trial. Lancet Respir Med. 2020;8(3):267-276.

146. Corral L, Bahamonde A, Delasrevillas F, et al. Glucocovid: A Controlled Trial of Methylprednisolone In Adults Hospitalized With Covid-19 Pneumonia. Medrxiv. 2020. doi:10.1101/2020.06.17.20133579
147. Ruiz-Irastorza G, Pijoan J, Bereciartua E, et al. Second-Week Methylprednisolone Pulses Improve Prognosis In Patients With Severe Coronavirus Disease 2019 Pneumonia: an Observational Comparative Study Using Routine Care Data. PLoS One. 2020;15 (9):E0239401.

148. Rahman O, Trigonis R, Craft M, et al. Corticosteroid Use In Severely Hypoxemic Covid-19 Patients: an Observational Cohort Analysis Of Dosing Patterns and Outcomes In The Early Phase Of The Pandemic. Medrxiv. 2020. doi:10.1101/ 2020.07.29.20164277

149. Colaneri M, Bogliolo L, Valsecchi P, et al. Tocilizumab for Treatment of Severe COVID-19 Patients: preliminary Results from SMAtteo COvid19 REgistry (SMACORE). Microorganisms. 2020;8(5):695.

150. Toniati P, Piva S, Cattalini $\mathrm{M}$, et al. Tocilizumab for The Treatment of Severe Covid-19 Pneumonia with Hyperinflammatory Syndrome and Acute Respiratory Failure: A Single-Center Study Of 100 Patients in Brescia, Italy. Autoimmun Rev. 2020;19(7):102568. doi:10.1016/J.Autrev.2020.102568

151. Sciascia S, Aprà F, Baffa A, et al. Pilot Prospective Open, Single-Arm Multicentre Study on Off-Label Use of Tocilizumab in Patients with Severe Covid-19. Clin Exp Rheumatol. 2020;38 (3):529-532.

152. Biran N, Ip A, Ahn J, et al. Tocilizumab Among Patients With Covid-19 In The Intensive Care Unit: A Multicentre Observational Study. Lancet Rheumatology. 2020;2(10):E603-E12.

153. Guaraldi G, Meschiari M, Cozzi-Lepri A. et al. Tocilizumab in Patients with Severe Covid-19: A Retrospective Cohort Study. Lancet Rheumatol. 2020;2(8):E474-E484. doi:10.1016/S26659913(20)30173-9

154. Moreno-Pérez O, Andres M, Leon-Ramirez J, et al. Experience with Tocilizumab in Severe Covid-19 Pneumonia After 80 Days of Follow-Up: A Retrospective Cohort Study. J Autoimmun. 2020;16:102523.

155. De Rossi N, Scarpazza C, Filippini C, et al. Early Use of Low Dose Tocilizumab in Patients with Covid-19: A Retrospective Cohort Study with A Complete Follow-Up. Eclinicalmedicine. 2020;25:100459. doi:10.1016/J.Eclinm.2020.100459

156. Somers E, Eschenauer G, Troost JP, et al. Tocilizumab for Treatment of Mechanically Ventilated Patients with Covid-19. Clin Infect Dis. 2020:Ciaa954. doi:10.1093/Cid/Ciaa954

157. Price C, Altice F, Shyr Y, et al. Tocilizumab Treatment for Cytokine Release Syndrome in Hospitalized Covid-19 Patients: survival and Clinical Outcomes. Chest. 2020;S0012-3692 (20):31670-31676. doi:10.1016/J.Chest.2020.06.006

158. Fernández-Ruiz M, López-Medrano $\mathrm{F}$. Tocilizumab for The Treatment of Adult Patients with Severe Covid-19 Pneumonia: A Single-Center Cohort Study. J Med Virol. 2020.

159. Luo P, Liu Y, Qiu L, Liu X, Liu D, Li J. Tocilizumab Treatment in Covid-19: A Single-Center Experience. J Med Virol. 2020;92 (7):814-818. doi:10.1002/Jmv.25801

160. Mastroianni A, Greco S, Apuzzo G, et al. Subcutaneous Tocilizumab Treatment in Patients with Severe Covid-19Related Cytokine Release Syndrome: an Observational Cohort Study. Eclinicalmedicine. 2020;24:100410. doi:10.1016/J. Eclinm.2020.100410

161. Klopfenstein T, Zayet S, Lohse A, et al. Tocilizumab Therapy Reduced Intensive Care Unit Admissions and/or Mortality In Covid-19 Patients. Med Mal Infect. 2020;50(5):397-400.

162. Gupta S, Wang W, Hayek S, et al. Association Between Early Treatment with Tocilizumab and Mortality Among Critically Ill Patients with Covid-19. JAMA Intern Med. 2020. doi:10.1001/ Jamainternmed.2020.6252

163. Lan S, Lai C, Huang H, Chang S. For Severe Covid-19: A Systematic Review and Meta-Analysis. Int $J$ Antimicrob Agents. 2020;56(3):10610'. 
164. Knorr J, Colomy V, Mauriello C, Ha S. Tocilizumab in Patients with Severe Covid-19: A Single-Center Observational Analysis. J Med Virol. 2020.

165. Kewan T, Covut F, Al-Jaghbeer M, Rose L, Gopalakrishna K, Akbik B. Tocilizumab For Treatment Of Patients With Severe Covid-19: A Retrospective Cohort Study. Eclinicalmedicine. 2020;24:100418. doi:10.1016/J.Eclinm.2020.100418

166. Campochiaro C, Della-Torre E, Cavalli G, et al. Efficacy and Safety of Tocilizumab in Severe Covid-19 Patients: A Single-Center Retrospective Cohort Study. Eur J Intern Med. 2020;76:43-49. doi:10.1016/J.Ejim.2020.05.021

167. Morena V, Milazzo L, Oreni L, et al. Off-Label Use of Tocilizumab for The Treatment Of Sars-Cov-2 Pneumonia In Milan, Italy. Eur J Intern Med. 2020;76:36-42. doi:10.1016/J. Ejim.2020.05.011

168. Rimland C, Morgan C, Bell G, et al. Clinical Characteristics and Early Outcomes In Patients With Covid-19 Treated With Tocilizumab At A United States Academic Center. Medrxiv. 2020. doi:10.1101/2020.05.13.20100404

169. Salvarani C, Dolci G, Massari M, et al. Effect of Tocilizumab Vs. Standard Care On Clinical Worsening In Patients Hospitalized With Covid-19 Pneumonia: A Randomized Clinical Trial. JAMA Intern Med. 2020. doi:10.1001/Jamainternmed.2020.6615

170. Tleyjeh IM, Kashour Z, Damlaj M, et al. Efficacy and safety of tocilizumab in COVID-19 patients: a living systematic review and meta-analysis. Clin Microbiol Infect. 2020;S1198-743X (20):30690. doi:10.1016/j.cmi.2020.10.036

171. Samad N, Sodunke TE, Banna HA, et al. Convalescent Plasma Therapy for Management of COVID-19: perspectives and Deployment in the Current Global Pandemic. Risk Manag Healthc Policy. 2020;13:2707-2728. doi:10.2147/RMHP.S281388

172. Duan K, Liu B, Li C, et al. Effectiveness of Convalescent Plasma Therapy In Severe Covid-19 Patients. Proc Natl Acad Sci USA. 2020;117(17):9490-9496. doi:10.1073/Pnas.2004168117

173. Rasheed A, Ftak D, Hashim H, Maulood MF. The Therapeutic Effectiveness of Convalescent Plasma Therapy On Treating Covid-19 Patients Residing In Respiratory Care Units In Hospitals In Baghdad, Iraq. Medrxiv. 2020. doi:10.1101/ 2020.06.24.20121905
174. Salazar E, Perez K, Ashraf M, et al. Treatment Of Coronavirus Disease 2019 (Covid-19) Patients With Convalescent Plasma. Am J Pathol. 2020;190(8):1680-1690. doi:10.1016/J.Ajpath.2020.05.014

175. Rabelo-Da-Ponte F, Silvello D, Scherer J, Ayala A, Klamt F. Convalescent Plasma Therapy In Patients With Severe Or Life-Threatening Covid-19: A Metadata Analysis. J Infect Dis. 2020;222(9):1575.

176. Agarwal A, Mukherjee A, Kumar G, et al. Convalescent Plasma in The Management Of Moderate Covid-19 In India: an Open-Label Parallel-Arm Phase II Multicentre Randomized Controlled Trial (Placid Trial). BMJ. 2020;371:M3939. doi:10.1136/Bmj.M3939

177. King A, Vail A, O'Leary C, et al. Anakinra in COVID-19: important considerations for clinical trials. Lancet Rheumatology. 2020. doi:10.1016/S2665-9913(20)30160-0

178. Cavalli G, De Luca G, Campochiaro C, et al. Interleukin-1 Blockade with High-Dose Anakinra In Patients With Covid-19, Acute Respiratory Distress Syndrome, and Hyperinflammation: A Retrospective Cohort Study. Lancet Rheumatol. 2020;2(6): E325-E331. doi:10.1016/S2665-9913(20)30127-2

179. Haque M. The COVID-19 pandemic - a global public health crisis: a brief overview regarding pharmacological interventions. Pesqui Bras Odontopediatria Clín Integr. 2020;20(supp1):e0146. https://doi.org/10.1590/pboci.2020.137

180. Singh TU, Parida S, Lingaraju MC, Kesavan M, Kumar D, Singh RK. Drug repurposing approach to fight COVID-19. Pharmacol Rep. 2020 Dec;72(6):1479-1508. doi:10.1007/s43440-020-001556. Epub 2020 Sep 5. PMID: 32889701; PMCID: PMC7474498.

181. Sultana J, Crisafulli S, Gabbay F, Lynn E, Shakir S, Trifirò G. Challenges for Drug Repurposing in the COVID-19 Pandemic Era. Front Pharmacol. 2020 Nov 6;11:588654-E331. doi:10.3389/ fphar.2020.588654. PMID: 33240091; PMCID: PMC7677570.
Infection and Drug Resistance

\section{Publish your work in this journal}

Infection and Drug Resistance is an international, peer-reviewed openaccess journal that focuses on the optimal treatment of infection (bacterial, fungal and viral) and the development and institution of preventive strategies to minimize the development and spread of resistance. The journal is specifically concerned with the epidemiology of antibiotic resistance and the mechanisms of resistance development and diffusion in both hospitals and the community. The manuscript management system is completely online and includes a very quick and fair peerreview system, which is all easy to use. Visit http://www.dovepress.com/ testimonials.php to read real quotes from published authors. 